\title{
Energy-efficiency maximization bidirectional direct and relay transmission
}

\author{
Caixia Cai ${ }^{1,2}$, Runhe Qiu ${ }^{1,2^{*}}$ (D), Xue-Qin Jiang ${ }^{1,2}$ and Yuyang Peng ${ }^{3}$
}

\author{
*Correspondence: \\ qiurh@dhu.edu.cn \\ 'College of Information Sciences \\ and Technology, Donghua \\ University, Renmin North, Shanghai \\ 201620, People's Republic of China \\ 2Engineering Research Center of \\ Digitized Textile \& Fashion \\ Technology, Ministry of Education, \\ Donghua University, Renmin North, \\ Shanghai 201620, People's Republic \\ of China \\ Full list of author information is \\ available at the end of the article
}

\begin{abstract}
Energy-efficient transmission (EET) has become a very important problem in wireless communication. Optimal power allocation (OPA) is one of the general methods to achieve EET. But when OPA is only considered in EET, it maybe cannot analyze EET problems accurately. This paper aims at analyzing energy-efficient bidirectional direct and relay transmission (RT) through joint optimization of transmit power (TP) and transmit time (TT) allocation. In RT, direct links (DLs) are existed. The EET problems are given with three optimization cases: (i) maximizing sum throughput (ST) to maximize energy efficiency (EE), in such case, optimal $\Pi T(\mathrm{OTT})$, optimal relay position (ORP), and outage probability analysis are given; (ii) minimizing total energy consumption (TEC) to maximize EE, in such case, optimal TP (OTP), OTT with gradient-descent algorithm, and influences of asymmetry and asymmetry transmission tasks are given; and (iii) maximizing ST and minimizing TEC simultaneously to maximize EE, in such case, Dinkelbach's algorithm and one-by-one optimal algorithm are given. Simulation results are presented to validate theoretical analysis. Results reveal that relay technique and DLs in RT can improve system's EE.
\end{abstract}

Keywords: Energy efficiency, Direct links, Optimal transmit time, Optimal transmit power, Optimal relay position

\section{Introduction}

With rapid development of wireless communication, traditional communication system cannot satisfy the requirement of green communication and spectrum resources shortage $[1,2]$. A large amount of interests have been focused on improving energy efficiency (EE) and spectral efficiency (SE). At the same time, lots of works have been done with different transmission techniques to improve them separately or simultaneously, such as cognitive radio [3, 4], orthogonal frequency division multiplexing (OFDM) [5], multi antenna technique [6], millimeter wave technique [7], simultaneous wireless information and power transfer (SWIPT) technique [8, 9], and non-orthogonal multiple access [10, 11]. Among all of these techniques, relay technique has attracted a lot of attention for achieving spatial diversity [12]. The major existing relay protocols are amplify-and-forward (AF) and decode-and-forward (DF). In AF relay protocol, relay node simply amplifies and forwards the received signal without any decoding operation [13]. Also, AF relay protocol is easy

(C) The Author(s). 2020 Open Access This article is licensed under a Creative Commons Attribution 4.0 International License which permits use, sharing, adaptation, distribution and reproduction in any medium or format, as long as you give appropriate credit to the original author(s) and the source, provide a link to the Creative Commons licence, and indicate if changes were made. The images or other third party material in this article are included in the article's Creative Commons licence, unless indicated otherwise in a credit line to the material. If material is not included in the article's Creative Commons licence and your intended use is not permitted by statutory regulation or exceeds the permitted use, you will need to obtain permission directly from the copyright holder. To view a copy of this licence, visit http://creativecommons.org/licenses/by/4.0/. 
for implementation since it only requires coarse synchronization [14]. Therefore, AF relay protocol is considered in this paper.

With relay technique, energy-efficient transmission (EET) can be achieved. Energyefficient resource allocation has been investigated in [15], which demonstrated that system EE can be maximized by scheduling optimal numbers of relay antennas and corresponding relay transmit power (TP). Optimal power allocation (OPA) scheme in a cooperative relaying system has been investigated in [16], which suggested that optimal global EE is strictly quasi-concave with regard to TP. With relay technique, spectral-efficient transmission (SET) can also be achieved. Optimal rate allocation scheme has been proposed in [17], and it found that system's error-free SET can be achieved. Energy-and-spectralefficient adaptive forwarding strategy for multi-hop device-to-device communications overlaying cellular networks has been proposed in [18], and it found that the higher EE and SE can be achieved.

However, only OPA has been considered in $[15,16]$ and only ideal network environment has been considered in $[17,18]$ to achieve EET. Actually, joint optimization of transmit time (TT) and TP allocation can be more accurately to measure system's energy consumption and more effectively to improve system's EE. Considering the joint optimization concern, energy-efficient relay-assisted cellular network has been studied in [19], which aimed at minimizing total power consumption through joint optimization of TT and TP allocation, and EE comparisons among direct transmission (DT), one-way relay transmission (OWRT), and two-way relay transmission (TWRT) with consideration of optimal TT (OTT) and optimal TP (OTP) have been discussed in [20]. It should be noted that the TWRT in [20] transmitted signal with the two-phase analog network coding (ANC) protocol. At the same time, in practical transmission systems, energy consumption does include not only TP, but also non-negligible circuit powers (CPs). In addition, the power amplifier (PA) efficiency is usually not ideal. The CP consumption and non-ideal PA efficiency are all for non-ideal network environment. Considering the non-ideal network environment concern, throughput optimal policies for energy harvesting transmitters with CP consumption have been studied in [21], and EE maximization of full-duplex (FD) two-way DF relay with PA efficiency and CP consumption has been discussed in [22]. While considering the above two concerns, EE analysis of relay systems with joint consideration of TP, TT, and CPs has been discussed in [23].

However, again, direct links (DLs) in relay transmission (RT) have not been considered in [19-23] with assumption of deeply fading channel. Actually, consideration of DLs that are existed in RT can achieve further SE performance gain, and some works have been done on it. For example, cellular communication scenario involving the coexistence of one-hop DT and two-hop relaying has been studied in [24], optimal source and relay design for multiuser MIMO AF relay communication system with DLs and imperfect channel state information (CSI) has been investigated in [25], EE optimization for a twohop AF relay network with DLs over Rayleigh fading channels has been considered in [26], RT considering DLs and aiming at maximizing EE has been discussed in [27], and the optimization for energy-efficient FD transmissions with DLs has been studied in our recent work [28]. As for the comparisons between the half-duplex and FD technique of the related works, they have been given in [28]. It has been shown that the FD technique can increase the $\mathrm{SE}$ and $\mathrm{EE}$ of the system, but only the minimizing total energy consumption (TEC) case has been discussed in [27, 28]. 
At the same time, optimal relay position (ORP) problem is important in RT for it influences the system performance. Although it has been showed that the ORP in a general communication system is the relay node located at the middle of two source nodes, it still can be a open problem. To the best of our knowledge, there are fewer works that investigate the ORP problem from the perspective of maximizing EE, i.e., the problem of joint optimization of relay station positions and relay stations serving range for maximizing EE has been discussed in [29].

Considering all of the above concerns and comparing with existing work in the literatures, whose characteristics are summarized in Table 1, this paper investigates the energy-efficient DT and RT problems. The main contributions of this paper can be summarized as follows:

- Firstly, to achieve a more pratical EET in this paper, PA efficiency, CP consumption, DLs in RT, and joint optimization of TT and TP allocation are considered simultaneously, rather than only considering parts of them in existing work. At the same time, ORP problem is investigated from the perspective of maximizing EE for the system EE can be improved with ORP [29]. This problem also has not been considered in the most of existing work.

- Secondly, to achieve a more comprehensive EE analysis in this paper, EET problems are given with three optimization cases, i.e., maximizing sum throughput (ST) to maximize EE, minimizing TEC to maximize EE, and maximizing ST and minimizing TEC simultaneously to maximize EE, rather than only considering minimizing TEC to maximize EE in [19] and [22, 23], or only considering maximizing ST to maximizing EE in [18] and [29, 30].

- Thirdly, with the three optimization cases: (1) OTT, ORP, and outage probability analysis are given with maximizing ST to maximize EE; (2) OTP, OTT with gradient-descent algorithm, and influences of asymmetry and asymmetry transmission tasks are given with minimizing TEC to maximize EE; (3) Dinkelbach's algorithm and one-by-one optimal algorithm are given with maximizing ST and minimizing TEC simultaneously to maximize EE.

The rest of this paper is organized as follows. Section 2 describes the system model. Section 3 provides the throughput analysis, energy consumption model, and problem formulation. Sections 4, 5, and 6 presents the three optimization cases to maximize EE. Section 7 gives simulation results and followed by conclusions in Section 8 .

\section{System model}

In this section, the models of bidirectional DT and RT are explained. In DT, there are two source nodes $S_{1}$ and $S_{2}$. In RT, there is also a relay node $R$ except for two source

Table 1 Existing literature comparison with this paper

\begin{tabular}{ll}
\hline Optimal transmit power (OTP) & {$[15-16],[19-23],[25-28]$, this paper } \\
Optimal transmit time (OTT) & {$[19-20],[22-23],[27-28]$, this paper } \\
Circuit power (CP) consumption & {$[15-16],[20-23],[26-28]$, this paper } \\
Power amplifier (PA) efficiency & {$[15],[20-23],[26-28]$, this paper } \\
Direct links (DLs) & {$[18],[24-28]$, this paper } \\
Optimal relay position (ORP) & {$[17],[27],[29-30]$, this paper } \\
\hline
\end{tabular}


nodes $S_{1}$ and $S_{2}$. At the same time, the relay node $R$ lies between two source nodes $S_{1}$ and $S_{2}$. To achieve a bidirectional transmission, it needs two time slots in DT, it needs four time slots in OWRT, and it needs three time slots in TWRT. The relay protocol is AF, and nodes' work mode is half-duplex. The links experience independent block Rayleigh fading and remain unchanged during one block. The following optimization and analysis are based on one block duration $T_{t}$. The transmission signal for the node $S_{1}$ is $x_{1}$ with variance $E\left\{x_{1}{ }^{2}\right\}=1$, and the transmission signal for the node $S_{2}$ is $x_{2}$ with variance $E\left\{x_{2}{ }^{2}\right\}=1$. The TP for the node $S_{1}$ is $P_{1}$, and the TP for the node $S_{2}$ is $P_{2}$. The channel gain between the nodes $S_{1}$ and $S_{2}$ is $h_{3}$, the channel gain between the nodes $S_{1}$ and $R$ is $h_{1}$, and the channel gain between the nodes $S_{2}$ and $R$ is $h_{2}$. At the same time, the channel gains between the same two nodes are all reciprocal, and the nodes have the full CSI. The noise at the node $S_{1}$ is $n_{1}$, the noise at the node $S_{2}$ is $n_{2}$, and the noise at the node $R$ is $n_{r}$. Meanwhile, the DLs between two source nodes $S_{1}$ and $S_{2}$ in RT are existed and they can be exploited to convey information [25]. The noises are zero-mean symmetric complex Gaussian vector with variance 1 . The system bandwidth is $W$. The receive signals are combined at two nodes $S_{1}$ and $S_{2}$ by maximum ratio combining (MRC) technique.

The DT model is shown in Fig. 1a. In the first time slot, source node $S_{1}$ transmits signal $x_{1}$ to node $S_{2}$, and the receive signal at node $S_{2}$ is $y_{2}^{d}=\sqrt{P_{1}} h_{3} x_{1}+n_{2}$. In the second time slot, source node $S_{2}$ transmits signal $x_{2}$ to node $S_{1}$, and the receive signal at node $S_{1}$ is $y_{1}^{d}=\sqrt{P_{2}} h_{3} x_{2}+n_{1}$.

The OWRT model is shown in Fig. 1b. In the first time slot, source node $S_{1}$ transmits signal $x_{1}$ to node $R$ and $S_{2}$, and the receive signals at node $R$ and $S_{2}$ are respectively $y_{1 r}^{o}=\sqrt{P_{1}} h_{1} x_{1}+n_{r}$ and $y_{2}^{o}=\sqrt{P_{1}} h_{3} x_{1}+n_{2}$. In the second time slot, relay node $R$ amplifies and forwards the receive signals $y_{1 r}^{o}$ to node $S_{2}$, and the receive signal at node $S_{2}$ is $y_{r 2}^{o}=\sqrt{P_{r 2}} h_{2} x_{1 r}+n_{2}$, where $P_{r 2}$ is relay node's TP in the second time slot of OWRT, $x_{1 r}$ is node $R$ amplifies and forwards signal in the second time slot of OWRT

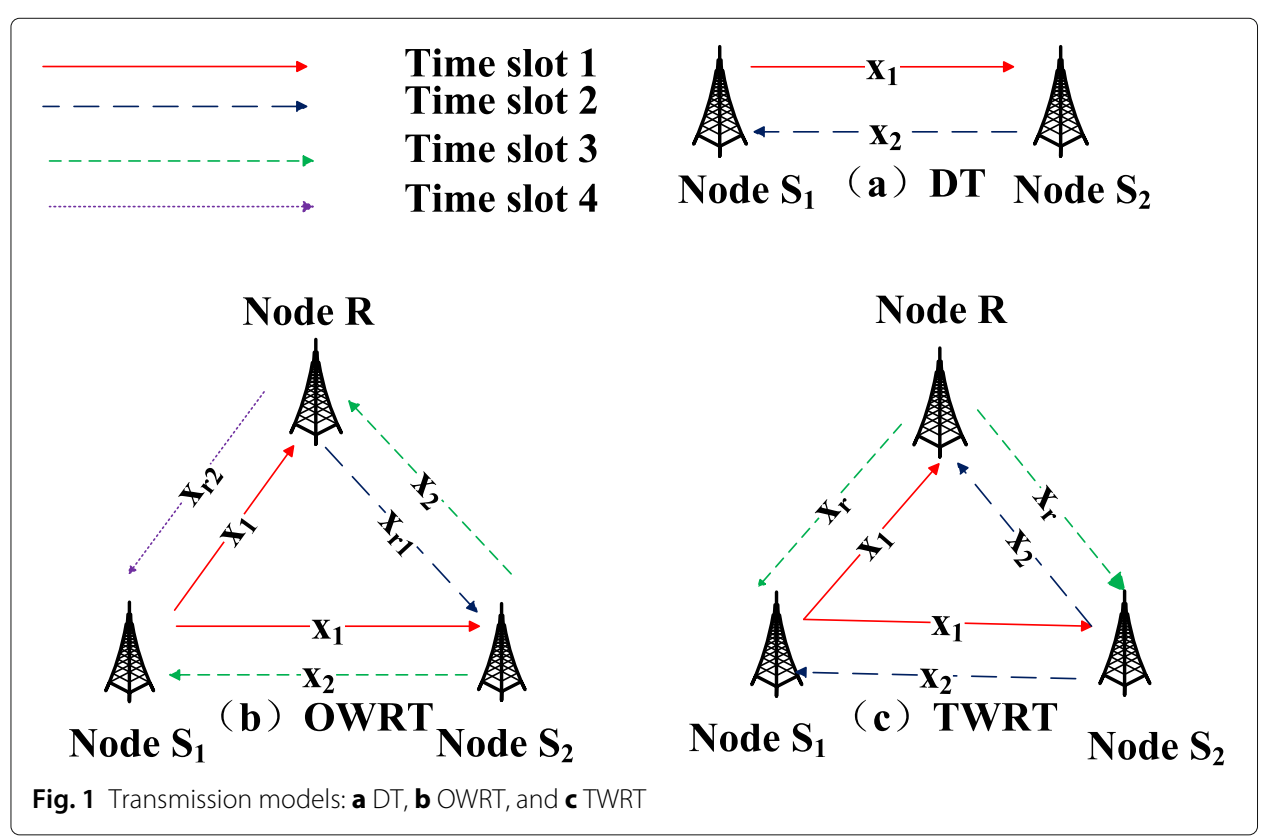


with $x_{1 r}=A_{1} y_{1 r}^{o}, A_{1}$ is amplify coefficients and $A_{1} \approx \frac{1}{\sqrt{P_{1}\left|h_{1}\right|^{2}}}$ [31]. In the third time slot, source node $S_{2}$ transmits signal $x_{2}$ to node $R$ and $S_{1}$, and the receive signals at relay node $R$ and $S_{1}$ are respectively $y_{2 r}^{o}=\sqrt{P_{2}} h_{2} x_{2}+n_{r}$ and $y_{1}^{o}=\sqrt{P_{2}} h_{3} x_{2}+n_{1}$. In the fourth time slot, relay node $R$ amplifies and forwards the receive signals $y_{2 r}^{o}$ to node $S_{1}$, and the receive signal at node $S_{1}$ is $y_{r 1}^{o}=\sqrt{P_{r 1}} h_{1} x_{2 r}+n_{1}$, where $P_{r 1}$ is relay node's TP in the fourth time slot of OWRT, $x_{2 r}$ is node $R$ amplifies and forwards signal in the fourth time slot of OWRT with $x_{2 r}=A_{2} y_{2 r}^{o}, A_{2}$ is amplify coefficients, and $A_{2} \approx \frac{1}{\sqrt{P_{2}\left|h_{2}\right|^{2}}}[31]$.

The TWRT model is shown in Fig. 1c, and it transmits signal with the three-phase ANC protocol. The three-phase ANC protocol is also called time division broadcast channel (TDBC) protocol as [31] suggested. In the first time slot, source node $S_{1}$ transmits signal $x_{1}$ to node $R$ and $S_{2}$, and the receive signals at node $R$ and $S_{2}$ are respectively $y_{1 r}^{t}=y_{1 r}^{o}$ and $y_{2}^{t}=y_{2}^{o}$. In the second time slot, source node $S_{2}$ transmits signal $x_{2}$ to node $R$ and $S_{1}$, and the receive signals at relay node $R$ and $S_{1}$ are respectively $y_{2 r}^{t}=y_{2 r}^{o}$ and $y_{1}^{t}=y_{1}^{o}$. In the third time slot, relay node $R$ broadcasts $x_{r}$ to nodes $S_{1}$ and $S_{1}$, where $x_{r}=\zeta_{1} y_{1 r}^{t}+\zeta_{2} y_{2 r}^{t}$ is node $R$ amplifies and forwards signal of TWRT. $\zeta_{1}$ and $\zeta_{2}$ are forward coefficients, where $\zeta_{1} \approx \sqrt{\frac{o_{1}}{P_{1}\left|h_{1}\right|^{2}}}$ and $\zeta_{2} \approx \sqrt{\frac{o_{2}}{P_{2}\left|h_{2}\right|^{2}}}$. $o_{1}$ and $o_{2}$ can be seemed as the signal combining factors, and they determine how relay node $R$ combines the two signals $y_{1 r}^{t}$ and $y_{2 r}^{t}$, where $0 \leq\left\{o_{1}, o_{2}\right\} \leq 1$ and $o_{1}+o_{2}=1$ [31]. The original receive signals of TWRT $y_{r 1}^{t}{ }^{\prime}$ and $y_{r 2}^{t}{ }^{\prime}$ can be expressed as $y_{r 1}^{t}{ }^{\prime}=\sqrt{P_{r}} h_{1} x_{r}+n_{1}$ and $y_{r 2}^{t}{ }^{\prime}=\sqrt{P_{r}} h_{2} x_{r}+n_{2}$, where $P_{r}$ is relay node's TP in TWRT. Since each of source node receives a copy of its own transmitted signal as interference, the signal transmitted from the other source node can be decoded after self-interference cancellation (SIC). Finally, the receive signals at the nodes $S_{1}$ and $S_{2}$ can be respectively expressed as $y_{r 1}^{t}=\sqrt{P_{r}} h_{1}\left(\zeta_{2} \sqrt{P_{2}} h_{2} x_{2}+\zeta_{1} n_{r}+\zeta_{2} n_{r}\right)+n_{1}$ and $y_{r 2}^{t}=\sqrt{P_{r}} h_{2}\left(\zeta_{1} \sqrt{P_{1}} h_{1} x_{1}+\zeta_{1} n_{r}+\zeta_{2} n_{r}\right)+n_{2}$.

\section{Throughput analysis, energy consumption model, and problem formulation}

In this section, throughput analysis is given first, followed by the energy consumption model and problem formulation.

\subsection{Throughput analysis}

In this subsection, throughput analysis is given. As we have suggested in the system model, one block duration is $T_{t}$ and within which a round of bidirectional transmission is accomplished [22].

In DT and OWRT, $T_{1}$ and $T_{2}$ can be used to represent TT from node $S_{1}$ to $S_{2}$ and from node $S_{2}$ to $S_{1}$, respectively. With throughput definition in [22], $y_{1}^{d}$, and $y_{2}^{d}$, throughput of DT can be given by $C_{d}=C_{d_{1}}+C_{d_{2}}$, where $C_{d}$ is ST of DT, $C_{d_{1}}=$ $T_{1} W \log _{2}\left(1+\gamma_{d_{1}}\right)$ is throughput at node $S_{2}$ of DT, and $C_{d_{2}}=T_{2} W \log _{2}\left(1+\gamma_{d_{2}}\right)$ is throughput at node $S_{1}$ of DT. $\gamma_{d_{1}}$ and $\gamma_{d_{2}}$ are signal-to-noise ratios (SNRs) at nodes $S_{2}$ and $S_{1}$ of DT, respectively. With noise variance is 1 , they can be given by $\gamma_{d_{1}}=P_{1}\left|h_{3}\right|^{2}$ and $\gamma_{d_{2}}=P_{2}\left|h_{3}\right|^{2}$.

With $y_{2}^{o}, y_{r 2}^{o}, y_{1}^{o}$, and $y_{r 1}^{o}$, throughput of OWRT can be given by $C_{o}=C_{o_{1}}+C_{o_{2}}$, where $C_{o}$ is ST of OWRT, $C_{o_{1}}=\frac{T_{1}}{2} W \log _{2}\left(1+\gamma_{o_{1}}\right)$ is throughput at node $S_{2}$ of OWRT, and $C_{o_{2}}=$ $\frac{T_{2}}{2} W \log _{2}\left(1+\gamma_{o_{2}}\right)$ is throughput at node $S_{1}$ of OWRT. The $1 / 2$ is due to two time slots in completing a transmission process in each direction. $\gamma_{o_{1}}$ and $\gamma_{o_{2}}$ are respectively SNRs 
at nodes $S_{2}$ and $S_{1}$ of OWRT, and they can be given by $\gamma_{o_{1}}=P_{1}\left|h_{3}\right|^{2}+\frac{A_{1}^{2} P_{1} P_{r 2}\left|h_{1}\right|^{2}\left|h_{2}\right|^{2}}{A_{1}^{2} P_{r 2}\left|h_{2}\right|^{2}+1}$ and $\gamma_{o_{2}}=P_{2}\left|h_{3}\right|^{2}+\frac{A_{2}^{2} P_{2} P_{r 1}\left|h_{2}\right|^{2}\left|h_{1}\right|^{2}}{A_{2}^{2} P_{r 1}\left|h_{1}\right|^{2}+1}$.

In TWRT, $T_{t w r}$ can be used to represent TT for the whole information exchange. With $y_{2}^{t}, y_{r 2}^{t}, y_{1}^{t}$, and $y_{r 1}^{t}$, throughput of TWRT can be given by $C_{t}=C_{t_{1}}+C_{t_{2}}$, where $C_{t}$ is ST of TWRT, $C_{t_{1}}=\frac{T_{t w r}}{3} W \log _{2}\left(1+\gamma_{t_{1}}\right)$ is throughput at node $S_{2}$ of TWRT, and $C_{t_{2}}=$ $\frac{T_{t w r}}{3} W \log _{2}\left(1+\gamma_{t_{2}}\right)$ is throughput at node $S_{1}$ of TWRT. The $1 / 3$ is due to equal TT is consumed in three time slots. $\gamma_{t_{1}}$ and $\gamma_{t_{2}}$ are respectively SNRs at nodes $S_{2}$ and $S_{1}$ of TWRT, and they can be given by $\gamma_{t_{1}}=P_{1}\left|h_{3}\right|^{2}+\frac{\zeta_{1}^{2} P_{1} P_{r}\left|h_{2}\right|^{2}\left|h_{1}\right|^{2}}{\left(\zeta_{1}^{2}+\zeta_{2}^{2}\right) P_{r}\left|h_{2}\right|^{2}+1}$ and $\gamma_{t_{2}}=P_{2}\left|h_{3}\right|^{2}+$ $\frac{\zeta_{2}^{2} P_{2} P_{r}\left|h_{1}\right|^{2}\left|h_{2}\right|^{2}}{\left(\zeta_{2}^{2}+\zeta_{2}^{2}\right) P_{r}\left|h_{1}\right|^{2}+1}$.

\subsection{Energy consumption model}

In this subsection, the TEC model is given. It should be noted that the TEC contains TPs and CPs. To reduce the TEC, the system may not use entire block duration $T_{t}$ for transmission. In each block, each node has three states: transmission, reception, and idle [32], which corresponding to $\mathrm{CP}$ consumptions of three states are respectively $P^{c t}, P^{c r}$, and $P^{c i}$. For node $S_{1}, S_{2}$, and $R$ all have three kinds of CP consumptions, we use subscript $l \in\{1,2, r\}$ to represent nodes $S_{1}, S_{2}$, and $R$, respectively. In such case, the CPs of three nodes with three states can be expressed as $P_{l}^{c t}, P_{l}^{c r}$, and $P_{l}^{c i}$, respectively. Also, the SIC in TWRT needs to consume CPs $P^{s i c}$. In such case, the CP consumptions for SIC of nodes $S_{1}$ and $S_{2}$ in TWRT can be expressed as $P_{1}^{s i c}$ and $P_{2}^{s i c}$, respectively. The non-ideal PA efficiency is $\mu$ and $\mu \geq 1$ [33].

From the above illustrations, the TEC is related to TPs, CPs, and PA efficiency, and TT can be known. Then, the TEC in DT is $E_{d}=T_{1}\left(\mu P_{1}+P_{d_{c 1}}-P_{D c i}\right)+T_{2}\left(\mu P_{2}+P_{d_{c 2}}-\right.$ $\left.P_{D c i}\right)+T_{t} P_{D c i}$, where $P_{d_{c 1}}=P_{1}^{c t}+P_{2}^{c r}$ and $P_{d_{c 2}}=P_{2}^{c t}+P_{1}^{c r}$ represent total CPs in the first and second time slots of DT, respectively. $T_{t}=T_{1}+T_{2}+T^{\prime}$ in DT and OWRT, where $T^{\prime}$ is the time in idle state. $P_{D c i}=P_{1}^{c i}+P_{2}^{c i}$ represents total idle CP in DT.

The TEC in OWRT is $E_{o}=T_{1}\left(\frac{\mu\left(P_{1}+P_{r 2}\right)}{2}+P_{o_{c 1}}-P_{R c i}\right)+$ $T_{2}\left(\frac{\mu\left(P_{2}+P_{r 1}\right)}{2}+P_{o_{c 2}}-P_{R c i}\right)+T_{t} P_{R c i}$, where $P_{o_{c 1}}=\frac{1}{2}\left(P_{1}^{c t}+P_{2}^{c r}+P_{r}^{c r}+P_{r}^{c t}+P_{2}^{c r}+P_{1}^{c i}\right)$ and $P_{o_{c 2}}=\frac{1}{2}\left(P_{2}^{c t}+P_{1}^{c r}+P_{r}^{c r}+P_{r}^{c t}+P_{1}^{c r}+P_{2}^{c i}\right)$ represent total CPs in the first and second two time slots of OWRT, respectively. $P_{R c i}=P_{1}^{c i}+P_{2}^{c i}+P_{r}^{c i}$ represents total idle $\mathrm{CP}$ in RT.

The TEC in TWRT is $E_{t}=T_{t w r}\left(\frac{\mu\left(P_{1}+P_{2}+P_{r}\right)}{3}+P_{t_{c 1}}+P_{t_{c 2}}+P_{t_{c 3}}-P_{R c i}\right)+$ $T_{t} P_{R c i}$, where $P_{t_{c 1}}=\frac{1}{3}\left(P_{1}^{c t}+P_{2}^{c r}+P_{r}^{c r}\right), P_{t_{c 2}}=\frac{1}{3}\left(P_{2}^{c t}+P_{1}^{c r}+P_{r}^{c r}\right)$, and $P_{t_{c 3}}=$ $\frac{1}{3}\left(P_{r}^{c t}+P_{1}^{c r}+P_{2}^{c r}+P_{1}^{s i c}+P_{2}^{s i c}\right)$ represent total CPs in the first, second, and third time slot, and $T_{t}=T_{t w r}+T^{\prime}$ in TWRT.

\subsection{Problem formulation}

In this subsection, EE definition and problem formulation are given. The EE in this paper is defined the same as [34], and it can be given as $\eta=\frac{C_{T}}{E_{T}}$, where $C_{T}$ is ST and $E_{T}$ is TEC. From the throughput analysis and energy consumption model, it can be known that both $E_{T}$ and $C_{T}$ have relations with TPs and TT, and we need to maximize it by maximizing $C_{T}$ and minimizing $E_{T}$ with OTP and OTT. Thus, energy-efficient optimization problem can be summarized as 


$$
\max _{C_{T}, E_{T}} \eta\left(C_{T}, E_{T}\right)
$$

For a more comprehensive analysis, we divide the optimization problem into three optimization cases. Firstly, maximizing $C_{T}$ to maximize $\eta$ when TPs are equally allocated as $[29,30]$. Secondly, minimizing $E_{T}$ to maximize $\eta$ when $C_{T}$ is constant as [22, 23]. Thirdly, maximizing $C_{T}$ and minimizing $E_{T}$ simultaneously to maximize $\eta$. The EE analysis of these three cases will be given in the following three sections.

\section{Maximize sum throughput}

Firstly, when TPs are equally allocated, which means $P_{1}=P_{2}=P_{r 1}=P_{r 2}=P_{r}=P$, we try to maximize $C_{T}$ with OTT to maximize $\eta$. TPs equally allocation is usually existed in practical system for simplicity, i.e., IS-95-CDMA system [35]. At the same time, the throughput is an important index that the communication systems want to pursue. Thus, the discussion about maximizing $C_{T}$ to maximize $\eta$ with TPs equally allocation maybe can effectively improve the EE of the IS-95-CDMA system under a certain situation.

\subsection{Optimal transmit time}

In this subsection, the OTT analysis is given. It should be noted that all the CPs are a fixed power cost from 0 to hundreds of $\mathrm{mW}$ [36]. However, although the CPs are a fixed power cost, they are concerned with TT. Then, we need to maximize $C_{T}$ through OTT to maximize $\eta$. In such case, Propositions 1 and 2 about OTT to maximize $\eta$ can be obtained.

Proposition 1 When $P_{1}=P_{2}=P_{r 1}=P_{r 2}=P_{r}=P$ and different nodes' $C P s$ for transmit, receive, and idle are the same, i.e., $P_{1}^{c t}=P_{2}^{c t}, P_{1}^{c r}=P_{2}^{c r}$, and $P_{1}^{c i}=P_{2}^{c i}$, the OTT in DT and OWRT to maximize $\eta$ is $T^{\prime}=0$.

Proof: With $P_{1}=P_{2}=P_{r 1}=P_{r 2}=P_{r}=P$ and different nodes' CPs are the same constants, $\gamma_{o_{1}}=\gamma_{o_{2}}$ and $P_{o_{c 1}}=P_{o_{c 2}}=P_{o_{c}}$ can be obtained. Then, $C_{o}=\frac{T_{1}+T_{2}}{2} W \log _{2}(1+$ $\left.\gamma_{o_{1}}\right)$ and $E_{o}=\left(\mu P+P_{o_{c}}\right)\left(T_{1}+T_{2}\right)+\left(T_{t}-T_{1}-T_{2}\right) P_{R c i}$ can be further obtained. In such case, EE of OWRT $\eta_{o}$ can be given as

$$
\begin{aligned}
\eta_{o} & =\frac{\frac{T_{1}+T_{2}}{2} W \log _{2}\left(1+\gamma_{o_{1}}\right)}{\left(\mu P+P_{o_{c}}\right)\left(T_{1}+T_{2}\right)+\left(T_{t}-T_{1}-T_{2}\right) P_{R c i}} \\
& \stackrel{a}{=} \frac{\frac{1}{2} W \log _{2}\left(1+\gamma_{o_{1}}\right)}{\mu P+P_{o_{c}}+\left(\frac{T_{t}}{T_{1}+T_{2}}-1\right) P_{R c i}} \\
& \stackrel{b}{=} \frac{W \log _{2}\left(1+\gamma_{o_{1}}\right)}{2\left(\mu P+P_{o_{c}}\right)} .
\end{aligned}
$$

The (a) step is for dividing $T_{1}+T_{2}$, and (b) step is for $T_{1}+T_{2}=T_{t}$ and $T^{\prime}=0$ to maximize $\eta_{o}$. The same conclusion of the OTT in DT is $T^{\prime}=0$ can also be obtained with $P_{1}=P_{2}=P_{r 1}=P_{r 2}=P_{r}=P$ and $P_{d_{c 1}}=P_{d_{c 2}}=P_{d_{c}}$ to maximize EE of DT $\eta_{d}$.

The proof is completed.

Proposition 2 When $P_{1}=P_{2}=P_{r 1}=P_{r 2}=P_{r}=P$, the OTT in TWRT to maximize $\eta$ is also $T^{\prime}=0$. 
Proof: With $P_{1}=P_{2}=P_{r 1}=P_{r 2}=P_{r}=P$, EE of TWRT $\eta_{t}$ can be given as

$$
\begin{aligned}
\eta_{t} & =\frac{\frac{T_{t w r}}{3} W\left(\log _{2}\left(1+\gamma_{t_{1}}\right)+\log _{2}\left(1+\gamma_{t_{2}}\right)\right)}{T_{t w r}\left(\frac{\mu\left(P_{1}+P_{2}+P_{r}\right)}{3}+P_{t_{c 1}}+P_{t_{c 2}}+P_{t_{c 3}}\right)+\left(T_{t}-T_{t w r}\right) P_{R c i}} \\
& \stackrel{a}{=} \frac{\frac{W}{3}\left(\log _{2}\left(1+\gamma_{t_{1}}\right)+\log _{2}\left(1+\gamma_{t_{2}}\right)\right)}{\frac{\mu\left(P_{1}+P_{2}+P_{r}\right)}{3}+\left(P_{t_{c 1}}+P_{t_{c 2}}+P_{t_{c 3}}\right)+\left(\frac{T_{t}}{T_{t w r}}-1\right) P_{R c i}} \\
& \stackrel{W}{=} \frac{W \log _{2}\left(1+\gamma_{t_{1}}+\gamma_{t_{2}}+\gamma_{t_{1}} \gamma_{t_{2}}\right)}{3 \mu P+3\left(P_{t_{c 1}}+P_{t_{c 2}}+P_{t_{c 3}}\right)} .
\end{aligned}
$$

The (a) step is for dividing $\frac{T_{t w r}}{3}$, and (b) step is for $T_{t w r}=T_{t}$ and $T^{\prime}=0$ to maximize $\eta_{t}$.

The proof is completed.

Remark 1: It should be noted that comparing with DT and OWRT, the OTT in TWRT to maximize $\eta_{t}$ has no constraints for CPs. At the same time, the OTT in it is always $T_{t w r}=T_{t}$ when TPs are not functions of TT even with unequally power allocation.

\subsection{Optimal relay position}

In this subsection, ORP analysis is given. Considering the Rayleigh fading channel, when three nodes are in a line and the distances of two source nodes between relay node are normalized by the distance between two source nodes $S_{1}$ and $S_{2}$, the channel gains can be expressed as $\left|h_{3}\right|^{2}=1,\left|h_{1}\right|^{2}=d^{-\alpha}, d \in(0,1)$, and $\left|h_{2}\right|^{2}=(1-d)^{-\alpha}$ [31], where $d$ is the distance between node $S_{1}$ and $R, \alpha$ is the path loss attenuation factor, and $\alpha \in[2,5]$ [37].

From (2), we can see that only $\gamma_{o_{1}}$ is needed to be maximized with ORP to maximize $\eta_{o}$ when $T^{\prime}=0$. Then, Proposition 3 about ORP in OWRT can be obtained.

Proposition 3 When $P_{1}=P_{2}=P_{r 1}=P_{r 2}=P_{r}=P$ and $T^{\prime}=0$, the ORP in OWRT to maximize $\eta_{o}$ is $d=0.5$.

Proof: With $P_{1}=P_{2}=P_{r 1}=P_{r 2}=P_{r}=P, T^{\prime}=0, A_{1}$, and channel gains, $\gamma_{o_{1}}$ can be reformulated as

$$
\gamma_{o_{1}}=P\left(1+\frac{d^{-\alpha}(1-d)^{-\alpha}}{(1-d)^{-\alpha}+d^{-\alpha}}\right) .
$$

With (4), when $d=0.5, \gamma_{o_{1}}$ can achieve the maximum for $\frac{d^{-\alpha}(1-d)^{-\alpha}}{(1-d)^{-\alpha}+d^{-\alpha}} \leq \frac{\sqrt{d^{-\alpha}(1-d)^{-\alpha}}}{2} \leq$ $\frac{(0.5)^{-\alpha}}{2}$. This means that $\eta_{o}$ is the maximum when $d=0.5$ for in such case $\gamma_{o_{1}}$ is the maximum.

The proof is completed.

From (3), we can also know that only the sum SNR of TWRT $\gamma_{t}=\gamma_{t_{1}}+\gamma_{t_{2}}+\gamma_{t_{1}} \gamma_{t_{2}}$ is needed to be maximized with ORP to maximize $\eta_{t}$ when $T^{\prime}=0$. However, it also should be noted that comparing with OWRT, the $o_{1}$ and $o_{2}$ will influence $\gamma_{t_{1}}$ and $\gamma_{t_{2}}$. Then, Proposition 4 about optimal $o_{1}, o_{2}$, and ORP in TWRT can be obtained.

Proposition 4 When $P_{1}=P_{2}=P_{r 1}=P_{r 2}=P_{r}=P$ and $T^{\prime}=0$, the optimal $o_{1}, o_{2}$, and ORP in TWRT to maximize $\eta_{t}$ are $o_{1}=o_{2}=0.5$ and $d$ is smaller.

Proof: Please see the Appendix: Proof of Proposition 4. 
The proof is completed.

\subsection{Outage probability analysis}

In this subsection, outage probability analysis is given for it is an important criterion to measure the performance of a link. With $C_{t h}$ to avoid disruption for a given throughput, then $C_{(\cdot)}<C_{t h}$ denotes the outage event, and $\operatorname{Pr}\left(C_{(\cdot)}<C_{t h}\right)$ denotes the outage probability.

Let $\gamma=P_{1}=P_{2}=P_{r 1}=P_{r 2}=P_{r}=P, C_{d}$ can be reformulated as

$$
C_{d}=\left(T_{1}+T_{2}\right) W \log _{2}\left(1+\gamma\left|h_{3}\right|^{2}\right) .
$$

It should be noted that bidirectional transmission system is in outage if any end-to-end transmission is in outage. The outage event of DT is given by $\min \left\{C_{d_{1}}, C_{d_{2}}\right\}<C_{t h}$, and it

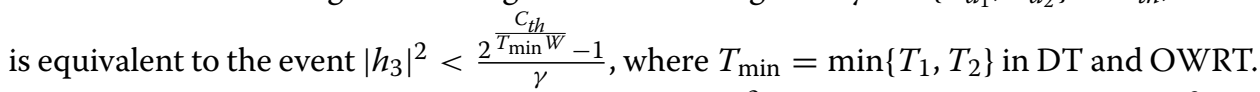
Statistically, the variances of channel gains $h_{l}$ are $\delta_{l}^{2}$. For Rayleigh fading, i.e., $\left|h_{l}\right|^{2}$ are exponentially distribution with parameter $\delta_{l}^{-2}$ [38], where subscript $l \in\{1,2,3\}$ represent different channel gains. Then, $\lambda_{1}=\delta_{1}^{-2}, \lambda_{2}=\delta_{2}^{-2}$, and $\lambda_{3}=\delta_{3}^{-2}$ can be obtained. Also, when $x$ tends to 0 , an equivalent infinitesimal approximation $1-e^{-x} \approx x$ can be obtained. With them, outage probability of DT $p_{d}^{\text {out }}$ can be calculated as

$$
\begin{aligned}
p_{d}^{\text {out }}\left(\gamma, C_{t h}\right) & =\operatorname{Pr}\left(\min \left\{C_{d_{1}}, C_{d_{2}}\right\}<C_{t h}\right) \\
& =\operatorname{Pr}\left(\left|h_{3}\right|^{2}<\frac{2^{\frac{C_{t h}}{T_{\min } W}}-1}{\gamma}\right) \\
& =1-e^{\left(-\frac{2^{\frac{C_{t h}}{T_{\min } W}}}{\gamma \delta_{3}^{2}}\right)} \approx \frac{1}{\delta_{3}^{2}} \cdot \frac{2^{\frac{C_{t h}}{T_{\min } W}}-1}{\gamma} .
\end{aligned}
$$

With $\gamma, C_{o}$ can be reformulated as

$$
C_{o}=\frac{T_{1}+T_{2}}{2} W \log _{2}\left(1+\gamma\left|h_{3}\right|^{2}+f\left(\gamma\left|h_{1}\right|^{2}, \gamma\left|h_{2}\right|^{2}\right)\right),
$$

where $f(x, y)=\frac{x y}{x+y}$. The outage event of OWRT is given by $\min \left\{C_{o_{1}}, C_{o_{2}}\right\}<C_{t h}$, and it is equivalent to the event

$$
\left|h_{3}\right|^{2}+\frac{1}{\gamma} f\left(\gamma\left|h_{1}\right|^{2}, \gamma\left|h_{2}\right|^{2}\right)<\frac{2^{\frac{2 C_{t h}}{T_{\min } W}}-1}{\gamma} .
$$

Assuming $\left|h_{3}\right|^{2}=s,\left|h_{1}\right|^{2}=u,\left|h_{2}\right|^{2}=v, m=\frac{1}{\gamma}, g(m)=\left(2^{\frac{2 C_{t h}}{T_{\min } W}}-1\right) m$, and according to [38], the following equation can be obtained as

$$
\lim _{m \rightarrow 0} \frac{1}{g^{2}(m)} \operatorname{Pr}\left(s+m f\left(\frac{u}{m}, \frac{v}{m}\right)<g(m)\right)=\frac{\lambda_{3}\left(\lambda_{1}+\lambda_{2}\right)}{2} .
$$

With it, we can further obtain the following equation as

$$
\lim _{m \rightarrow 0} \operatorname{Pr}\left(s+m f\left(\frac{u}{m}, \frac{v}{m}\right)<g(m)\right)=\frac{g^{2}(m) \lambda_{3}\left(\lambda_{1}+\lambda_{2}\right)}{2} .
$$


Finally, the outage probability of OWRT $p_{o}^{\text {out }}$ can be calculated as

$$
\begin{aligned}
p_{o}^{\text {out }}\left(\gamma, C_{t h}\right) & =\operatorname{Pr}\left(\min \left\{C_{o_{1}}, C_{o_{2}}\right\}<C_{t h}\right) \\
& =\operatorname{Pr}\left(s+\frac{1}{\gamma} f\left(\gamma\left|h_{1}\right|^{2}, \gamma\left|h_{2}\right|^{2}\right)<\frac{2^{\frac{2 C_{t h}}{T_{\min } W}}-1}{\gamma}\right) \\
& \approx\left(\frac{1}{2 \delta_{3}^{2}} \cdot \frac{\delta_{1}^{2}+\delta_{2}^{2}}{\delta_{1}^{2} \cdot \delta_{2}^{2}}\right)\left(\frac{2^{\frac{2 C_{t h}}{T_{\min } W}}-1}{\gamma}\right)^{2} .
\end{aligned}
$$

With $\gamma, C_{t}$ can be reformulated as

$$
\begin{aligned}
C_{t}= & \frac{T_{t w r}}{3} W \log _{2}\left(1+\gamma\left|h_{3}\right|^{2}+f_{1}\left(\gamma\left|h_{1}\right|^{2}, o_{1} \gamma\left|h_{2}\right|^{2}\right)\right) \\
& +\frac{T_{t w r}}{3} W \log _{2}\left(1+\gamma\left|h_{3}\right|^{2}+f_{2}\left(\gamma\left|h_{2}\right|^{2}, o_{2} \gamma\left|h_{1}\right|^{2}\right)\right),
\end{aligned}
$$

where $f_{1}(x, y)=\frac{x y}{x+y+o_{2} \gamma\left|h_{1}\right|^{2}}$ and $f_{2}(x, y)=\frac{x y}{x+y+o_{1} \gamma\left|h_{2}\right|^{2}}$. With $o_{1}=o_{2}=0.5$, the outage event of TWRT is given by $\min \left\{C_{t_{1}}, C_{t_{2}}\right\}<C_{t h}$, and it is equivalent to the event

$$
\left|h_{3}\right|^{2}+\frac{1}{\gamma} f_{1}\left(\gamma\left|h_{1}\right|^{2}, \gamma\left|h_{2}\right|^{2}\right)<\frac{2^{\frac{3 C_{t h}}{T_{t w r} W}}-1}{\gamma},
$$

where $f_{1}(x, y)=\frac{x y}{3 x+y}=\frac{1}{3} \cdot \frac{3 x y}{3 x+y}=\frac{1}{3} f(3 x, y)$. The above equivalent event give us an example when $C_{t_{1}} \leq C_{t_{2}}$ to get the outage probability of TWRT and we can use the same method to get the outage probability of TWRT when $C_{t_{1}}>C_{t_{2}}$. Assuming $3\left|h_{1}\right|^{2}=$ $w, h(m)=\left(2^{\frac{3 C_{t h}}{T_{t w r} W}}-1\right) m$, and according to [38], the following equation can also be obtained as

$$
\lim _{m \rightarrow 0} \frac{1}{h^{2}(m)} \operatorname{Pr}\left(s+\frac{1}{3} m f\left(\frac{w}{m}, \frac{v}{m}\right)<h(m)\right)=\frac{\lambda_{3}\left(3 \lambda_{1}+\lambda_{2}\right)}{6} .
$$

With it, we can further obtain the following equation as

$$
\lim _{m \rightarrow 0} \operatorname{Pr}\left(s+\frac{1}{3} m f\left(\frac{w}{m}, \frac{v}{m}\right)<h(m)\right)=\frac{h^{2}(m) \lambda_{3}\left(3 \lambda_{1}+\lambda_{2}\right)}{6} .
$$

Finally, the outage probability of TWRT $p_{t}^{\text {out }}$ can be calculated as

$$
\begin{aligned}
p_{t}^{\text {out }}\left(\gamma, C_{t h}\right) & =\operatorname{Pr}\left(\min \left\{C_{t_{1}}, C_{t_{2}}\right\}<C_{t h}\right) \\
& =\operatorname{Pr}\left(s+\frac{1}{3 \gamma} f\left(3 \gamma\left|h_{1}\right|^{2}, \gamma\left|h_{2}\right|^{2}\right)<\frac{2^{\frac{3 C_{t h}}{T_{t w r} W}}-1}{\gamma}\right) \\
& \approx\left(\frac{1}{6 \delta_{3}^{2}} \cdot \frac{\delta_{1}^{2}+3 \delta_{2}^{2}}{\delta_{1}^{2} \cdot \delta_{2}^{2}}\right)\left(\frac{2^{\frac{3 C_{t h}}{T_{t w r} W}}-1}{\gamma}\right)^{2} .
\end{aligned}
$$

With the same method to get (16), we can know that when $C_{t_{1}}>C_{t_{2}}$, the outage probability of TWRT $p_{t}^{\text {out }}$ is $p_{t}^{\text {out }}\left(\gamma, C_{t h}\right)=\operatorname{Pr}\left(\min \left\{C_{t_{1}}, C_{t_{2}}\right\}<C_{t h}\right) \approx\left(\frac{1}{6 \delta_{3}^{2}} \cdot \frac{3 \delta_{1}^{2}+\delta_{2}^{2}}{\delta_{1}^{2} \cdot \delta_{2}^{2}}\right)\left(\frac{2^{\frac{3 C_{t h}}{T_{t w r} W}}-1}{\gamma}\right)^{2}$.

\section{Minimize total energy consumption}

Secondly, when $C_{T}$ is constant with the assumption of $C_{1}=\beta C_{T}$ and $C_{2}=(1-\beta) C_{T}$, we try to minimize $E_{T}$ with OTT and OTP to maximize $\eta$. The $\beta \in(0,1)$ is the transmission task distribution factor. $C_{1}$ and $C_{2}$ are the minimum transmission tasks at two directions 
[22]. Minimizing TEC is one of the widely used methods to achieve green communication [1]. At the same time, the OPA has been widely studied in many practical wireless systems, i.e., with OPA to achieve EET of cellular system [19] and with OPA to maximize the average throughput and EE of energy harvesting system [21]. Thus, the discussion about the minimizing $E_{T}$ to maximize $\eta$ with joint optimization of TT and TP allocation maybe can effectively improve the EEs of the cellular and energy harvesting systems.

\subsection{Energy-efficient direct transmission}

In this subsection, the problem with minimizing $E_{d}$ to maximize $\eta_{d}$ is given. With the requirements of $C_{1}, C_{2}$, maximum TP $P_{t}^{\max }$, and total TT $T_{t}$, EET in DT can be given as follows:

$$
\begin{aligned}
\min _{P_{1}, P_{2}, T_{1}, T_{2}} & E_{d} \\
\text { s.t. } & 0 \leq\left\{P_{1}, P_{2}\right\} \leq P_{t}^{\max }, 0 \leq\left\{T_{1}, T_{2}\right\}, \\
& T_{1}+T_{2} \leq T_{t}, C_{d_{1}} \geq C_{1}, \text { and } C_{d_{2}} \geq C_{2} .
\end{aligned}
$$

Through throughputs, the TPs can be expressed as functions of TT, then the minimum TECs can be obtained with only optimizing TT. For throughputs are derived from Shannon capacity formula, which means the maximum achievable throughput can be obtained under given TPs, thus, the TPs derived through throughputs are also the minimum which can support the required transmission tasks.

With $C_{d_{1}}=C_{1}, 2^{\frac{C_{1}}{T_{1} W}}-1=\gamma_{d_{1}}$ can be obtained. At the same time, with $C_{d_{2}}=C_{2}$, $2^{\frac{C_{2}}{T_{2} W}}-1=\gamma_{d_{2}}$ can be obtained. Then through $\gamma_{d_{1}}=P_{1}\left|h_{3}\right|^{2}$ and $\gamma_{d_{2}}=P_{2}\left|h_{3}\right|^{2}$, the minimum TPs in DT can be obtained as

$$
P_{1}^{\mathrm{opt}}=\frac{\gamma_{d_{1}}}{\left|h_{3}\right|^{2}}, \quad P_{2}^{\mathrm{opt}}=\frac{\gamma_{d_{2}}}{\left|h_{3}\right|^{2}} .
$$

It can be seen that the optimal $P_{1}^{\text {opt }}$ and $P_{2}^{\text {opt }}$ are respectively increasing functions of $\gamma_{d_{1}}$ and $\gamma_{d_{2}}$; thus, they are respectively decreasing functions of $T_{1}$ and $T_{2}$. As $T_{1}$ and $T_{2}$ decrease, the optimal $P_{1}^{\mathrm{opt}}$ and $P_{2}^{\mathrm{opt}}$ increase, and they may achieve $P_{t}^{\max }$. To simplify the analysis, we only consider all the TPs that will not achieve $P_{t}^{\max }$ situation, and the TPs that achieve $P_{t}^{\max }$ situation can be seen in our recent work [27].

With (18), the (17) can be reformulated into only optimizing TT problem and it can be given by

$$
\begin{aligned}
& \min _{T_{1}, T_{2}} T_{1} \mu \frac{\gamma_{d_{1}}}{\left|h_{3}\right|^{2}}+T_{1}\left(P_{d_{c 1}}-P_{D c i}\right)+T_{2} \mu \frac{\gamma_{d_{2}}}{\left|h_{3}\right|^{2}}+T_{2}\left(P_{d_{c 2}}-P_{D c i}\right)+T_{t} P_{D c i} \\
& \text { s.t. } 0 \leq\left\{T_{1}, T_{2}\right\} \text { and } T_{1}+T_{2} \leq T_{t} .
\end{aligned}
$$

In (19), the term $T_{t} P_{D c i}$ is independent of TT, and the terms $E_{d_{1}}=T_{1} \mu \frac{\gamma_{d_{1}}}{\left|h_{3}\right|^{2}}+T_{1}\left(P_{d_{c 1}}-\right.$ $\left.P_{D c i}\right)$ and $E_{d_{2}}=T_{2} \mu \frac{\gamma_{d_{2}}}{\left|h_{3}\right|^{2}}+T_{2}\left(P_{d_{c 2}}-P_{D c i}\right)$ are only concerned with $T_{1}$ and $T_{2}$, respectively. Then, the second order derivative (SEC) $E_{d_{1}}^{\prime \prime}\left(T_{1}\right)=\frac{{ }^{\frac{C_{1}}{T_{1} W}} \mu(\ln 2)^{2} C_{1}^{2}}{W^{2}\left|h_{3}\right|^{2} T_{1}^{3}}$ and $E_{d_{2}}^{\prime \prime}\left(T_{2}\right)=$ $\frac{2^{\frac{C_{2}}{T_{2} W}} \mu(\ln 2)^{2} C_{2}^{2}}{W^{2}\left|h_{3}\right|^{2} T_{2}^{3}}$ can be obtained.

With $E_{d_{1}}^{\prime \prime}\left(T_{1}\right) \geq 0$ and $E_{d_{2}}^{\prime \prime}\left(T_{2}\right) \geq 0, E_{d_{1}}$ and $E_{d_{2}}$ that are respectively convex functions of $T_{1}$ and $T_{2}$ can be known. Therefore, the objective function is convex with respect to $T_{1}$ and $T_{2}$ for the addition of two convex functions is still a convex function [39]. The 


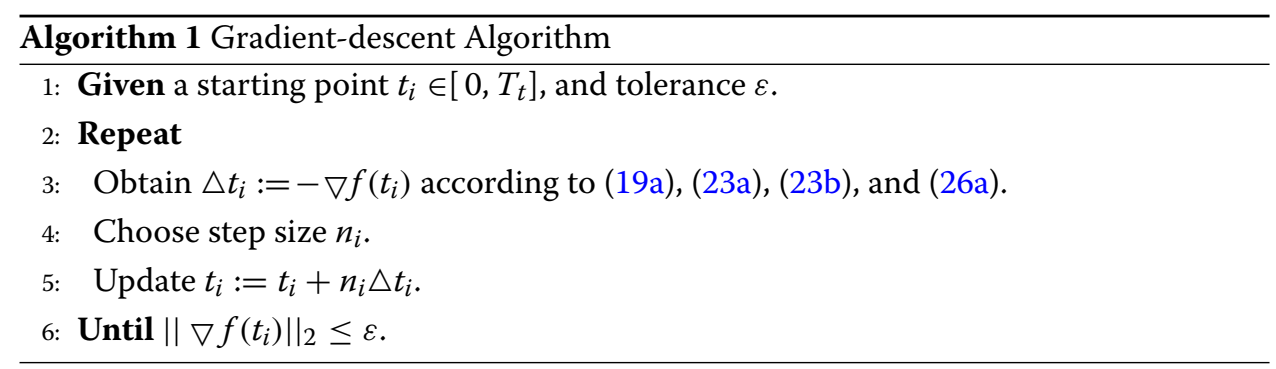

convex functions can be solved using standard solvers, such as inter-point methods, Newton method, and gradient-descent method. For the fast convergence of gradient-descent algorithm [39], we use it in this paper. For each $t_{i}$, the gradient-descent algorithm can be summarized as Algorithm 1.

In Algorithm 1, we give an initial point $t_{i} \in\left[0, T_{t}\right]$. Then, it iteratively moves toward lower values of the functions by taking steps in direction of negative gradient $-\nabla f\left(t_{i}\right)$ with chosen step size $n_{i}$, and $-\nabla f\left(t_{i}\right)$ can be obtained according to (19a), (23a), (23b), and (26a). Eventually, Algorithm 1 converges to the minimum for the functions are convex.

\subsection{Energy-efficient one-way relay transmission}

In this subsection, with minimizing $E_{o}$ to maximize $\eta_{o}$ problem is given. The EET in OWRT can also be given under the requirements of $C_{1}, C_{2}, P_{t}^{\max }$, and $T_{t}$. Observing $E_{o}$, it can be found that the terms $E_{o_{1}}=T_{1}\left(\frac{\mu\left(P_{1}+P_{r 2}\right)}{2}+P_{o_{c 1}}-P_{R c i}\right)$ and $E_{o_{2}}=$ $T_{2}\left(\frac{\mu\left(P_{2}+P_{r 1}\right)}{2}+P_{o_{c 2}}-P_{R c i}\right)$ are respectively only concerned with $T_{1}$ and $T_{2}$, and the term $T_{t} P_{R c i}$ is independent of TT. To make a further observation, it can be found that $\gamma_{o_{1}}$ is not a function of $P_{2}$ and $P_{r 1}$, while $\gamma_{o_{2}}$ is not a function of $P_{1}$ and $P_{r 2}$. Therefore, the optimization problem in OWRT can be equivalently reformulated into two subproblems as

$$
\begin{aligned}
\min _{P_{1}, P_{r 2}, T_{1}} & E_{o_{1}}=T_{1}\left(\frac{\mu\left(P_{1}+P_{r 2}\right)}{2}+P_{o_{c 1}}-P_{R c i}\right) \\
\text { s.t. } & 0 \leq\left\{P_{1}, P_{r 2}\right\} \leq P_{t}^{\max }, 0 \leq T_{1} \leq T_{t}, \text { and } C_{o_{1}} \geq C_{1}, \\
\min _{P_{2}, P_{r 1}, T_{2}} & E_{o_{2}}=T_{2}\left(\frac{\mu\left(P_{2}+P_{r 1}\right)}{2}+P_{o_{c 2}}-P_{R c i}\right) \\
\text { s.t. } 0 & \leq\left\{P_{2}, P_{r 1}\right\} \leq P_{t}^{\max }, 0 \leq T_{2} \leq T_{t} \text {, and } C_{o_{2}} \geq C_{2} .
\end{aligned}
$$

With $C_{o_{1}}=C_{1}, 2^{\frac{2 C_{1}}{T_{1} W}}-1=\gamma_{o_{1}}$ can be obtained. With $\gamma_{o_{1}}=P_{1}\left|h_{3}\right|^{2}+\frac{A_{1}^{2} P_{1} P_{r 2}\left|h_{1}\right|^{2}\left|h_{2}\right|^{2}}{A_{1}^{2} P_{r_{2}}\left|h_{2}\right|^{2}+1}$, $P_{r 2}$ can be obtained as $P_{r 2}=\frac{\left(\gamma_{o_{1}}-P_{1}\left|h_{3}\right|^{2}\right) P_{1}\left|h_{1}\right|^{2}}{\left|h_{2}\right|^{2}\left(P_{1} h_{13}-\gamma_{o_{1}}\right)}=f\left(P_{1}\right)$. With it, the TP in (20a) can be represented as $P_{1}+P_{r 2}=P_{1}+f\left(P_{1}\right)$. By setting the derivative of $P_{1}+f\left(P_{1}\right)$ to zero and with the help of its SEC, the optimal $P_{1}^{\mathrm{opt}}$ and $P_{r 2}^{\mathrm{opt}}$ with minimizing $P_{1}+P_{r 2}$ can be obtained as

$$
\begin{aligned}
& P_{1}^{\mathrm{opt}}=\frac{\left(c_{2}+\left(c_{1} c_{2}\right)^{\frac{1}{2}}\right) \gamma_{o_{1}}}{h_{13} c_{2}}, \\
& P_{r 2}^{\mathrm{opt}}=\frac{\left(c_{1} c_{2}-c_{1}\left|h_{1}\right|^{2}\left|h_{3}\right|^{2}+\left|h_{1}\right|^{2} h_{31}\left(c_{1} c_{2}\right)^{\frac{1}{2}}\right) \gamma_{o_{1}}}{\left|h_{2}\right|^{2} h_{13}^{2}\left(c_{1} c_{2}\right)^{\frac{1}{2}}},
\end{aligned}
$$


where $c_{1}=\left|h_{1}\right|^{4}, c_{2}=\left(\left|h_{2}\right|^{2}\left|h_{3}\right|^{2}+\left|h_{1}\right|^{2} h_{32}\right), h_{32}=\left|h_{2}\right|^{2}-\left|h_{3}\right|^{2}, h_{31}=\left|h_{1}\right|^{2}-\left|h_{3}\right|^{2}$, and $h_{13}=\left|h_{1}\right|^{2}+\left|h_{3}\right|^{2}$.

Following the same solving procedure as (20a), the optimal $P_{2}^{\mathrm{opt}}$ and $P_{r 1}^{\mathrm{opt}}$ with minimizing $P_{2}+P_{r 1}$ can be obtained as

$$
\begin{aligned}
& P_{2}^{\mathrm{opt}}=\frac{\left(c_{4}+\left(c_{3} c_{4}\right)^{\frac{1}{2}}\right) \gamma_{o_{2}}}{h_{23} c_{4}}, \\
& P_{r 1}^{\mathrm{opt}}=\frac{c_{3} \gamma_{o_{2}}\left(c_{4}-\left|h_{2}\right|^{2}\left|h_{3}\right|^{2}\right)+\left|h_{2}\right|^{2} h_{32}\left(c_{3} c_{4}\right)^{\frac{1}{2}} \gamma_{o_{2}}}{\left|h_{1}\right|^{2} h_{23}^{2}\left(c_{3} c_{4}\right)^{\frac{1}{2}}},
\end{aligned}
$$

where $2^{\frac{2 C_{2}}{T_{2} W}}-1=\gamma_{o_{2}}, c_{3}=\left|h_{2}\right|^{4}, c_{4}=\left(\left|h_{1}\right|^{2}\left|h_{3}\right|^{2}+\left|h_{2}\right|^{2} h_{31}\right)$, and $h_{23}=\left|h_{2}\right|^{2}+\left|h_{3}\right|^{2}$.

With (21) and (22), the (20) can be reformulated as

$$
\begin{aligned}
& \min _{T_{1}} E_{o_{1}}=T_{1}\left(\mu \frac{\left(\sqrt{c_{1}}+\sqrt{c_{2}}\right)^{2} \gamma_{o_{1}}}{2\left|h_{2}\right|^{2} h_{13}^{2}}+P_{o_{c 1}}-P_{R c i}\right) \\
& \text { s.t. } 0 \leq T_{1} \leq T_{t}, \\
& \min _{T_{2}} E_{o_{2}}=T_{2}\left(\mu \frac{\left(\sqrt{c_{3}}+\sqrt{c_{4}}\right)^{2} \gamma_{o_{2}}}{2\left|h_{1}\right|^{2} h_{23}^{2}}+P_{o_{c 2}}-P_{R c i}\right) \\
& \text { s.t. } 0 \leq T_{2} \leq T_{t},
\end{aligned}
$$

where $P_{1}^{\mathrm{opt}}+P_{r 2}^{\mathrm{opt}}=\frac{\left(\sqrt{c_{1}}+\sqrt{c_{2}}\right)^{2} \gamma_{o_{1}}}{\left|h_{2}\right|^{2} h_{13}^{2}}$, and $P_{2}^{\mathrm{opt}}+P_{r 1}^{\mathrm{opt}}=\frac{\left(\sqrt{c_{3}}+\sqrt{c_{4}}\right)^{2} \gamma_{o_{2}}}{\left|h_{1}\right|^{2} h_{23}^{2}}$. Then, the SEC

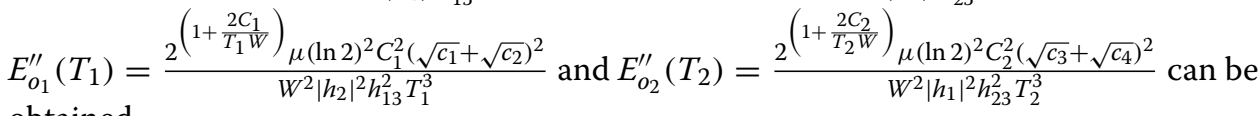
obtained.

With $E_{o_{1}}^{\prime \prime}\left(T_{1}\right) \geq 0$ and $E_{o_{2}}^{\prime \prime}\left(T_{2}\right) \geq 0, E_{o_{1}}$ and $E_{o_{2}}$ that are respectively convex functions of $T_{1}$ and $T_{2}$ can be known. Then, the problems can also be solved with Algorithm 1 as DT. It should be noted that the OTT in DT and OWRT should meet the requirement of $T_{1}^{\mathrm{opt}}+T_{2}^{\mathrm{opt}} \leq T_{t}$. If $T_{1}^{\mathrm{opt}}+T_{2}^{\mathrm{opt}}<T_{t}$, then we get $T_{1}^{\mathrm{opt}}$ and $T_{2}^{\mathrm{opt}}$ as the final OTT. Or if $T_{1}^{\text {opt }}+T_{2}^{\text {opt }}=T_{t}$, once $T_{1}^{\text {opt }}$ has been obtained, we can get $T_{2}^{\text {opt }}=T_{t}-T_{1}^{\text {opt }}$ through a scalar searching [23].

\subsection{Energy-efficient two-way relay transmission}

In this subsection, with minimizing $E_{t}$ to maximize $\eta_{t}$ problem is given. The EET in TWRT can also be given under the requirements of $C_{1}, C_{2}, P_{t}^{\max }$, and $T_{t}$. At the same time, $P_{1}=P_{2}=P$ in TWRT can be assumed as [31]. In such situation, the EET in TWRT can be given as follows:

$$
\begin{aligned}
\min _{P, P_{r}, T_{t w r}} & E_{t} \\
\text { s.t. } 0 & \leq\left\{P, P_{r}\right\} \leq P_{t}^{\max }, 0 \leq T_{t w r} \leq T_{t}, C_{t_{1}} \geq C_{1} \text {, and } C_{t_{2}} \geq C_{2} .
\end{aligned}
$$

Following the same solving procedure as (20a), the optimal $P^{\text {opt }}$ and $P_{r}^{\text {opt }}$ with minimizing $2 P+P_{r}$ can be obtained as

$$
\begin{aligned}
P^{\mathrm{opt}} & =\frac{B \gamma_{t_{1}}}{c_{7} c_{8}}, \\
P_{r}^{\mathrm{opt}} & =\frac{A B \gamma_{t_{1}}}{c_{8}^{2}\left(c_{6} c_{7}\left(c_{5}+c_{6}\right)\right)^{\frac{3}{2}}},
\end{aligned}
$$


where $2^{\frac{3 C_{1}}{T_{\text {twr }} W}}-1=\gamma_{t_{1}}, c_{5}=\left|h_{1}\right|^{2} o_{2}, c_{6}=\left|h_{2}\right|^{2} o_{1}, c_{7}=2\left|h_{2}\right|^{4} o_{1}+2\left|h_{1}\right|^{2}\left|h_{3}\right|^{2} o_{2}+$ $2\left|h_{2}\right|^{2}\left|h_{3}\right|^{2} o_{1}-\left|h_{2}\right|^{2}\left|h_{3}\right|^{2}, c_{8}=\left|h_{2}\right|^{4} o_{1}+\left|h_{1}\right|^{2}\left|h_{3}\right|^{2} o_{2}+\left|h_{2}\right|^{2}\left|h_{3}\right|^{2} o_{1}, A=\left|h_{2}\right|^{2} c_{6}\left(c_{5}+\right.$ $\left.c_{6}\right)\left(c_{6} c_{7}-\left|h_{3}\right|^{2}\left(c_{6} c_{7}\left(c_{5}+c_{6}\right)\right)^{\frac{1}{2}}\right)$, and $B=c_{7}\left(c_{5}+c_{6}\right)+\left|h_{2}\right|^{2}\left(c_{6} c_{7}\left(c_{5}+c_{6}\right)\right)^{\frac{1}{2}}$.

With (25), the (24) can be reformulated as

$$
\begin{aligned}
\min _{T_{t w r}} E_{t} & =T_{t w r}\left(\frac{\mu C \gamma_{t_{1}}}{3}+P_{t_{c 1}}+P_{t_{c 2}}+P_{t_{c 3}}-P_{R c i}\right)+T_{t} P_{R c i} \\
\text { s.t. } 0 & \leq T_{t w r} \leq T_{t},
\end{aligned}
$$

where $C=\frac{2 P^{\mathrm{opt}}+P_{r}^{\mathrm{opt}}}{\gamma_{t_{1}}}=\frac{\left|h_{2}\right|^{2} c_{6}\left(2 o_{1} h_{23}+h_{32}\right)+c_{5}\left(c_{7}+2 o_{1}\left|h_{2}\right|^{2}\left|h_{3}\right|^{2}\right)}{c_{8}^{2}}+\frac{2\left|h_{2}\right|^{2}\left(c_{6} c_{7}\left(c_{5}+c_{6}\right)\right)^{\frac{1}{2}}}{c_{8}^{2}}$. Then, the SEC $E_{t}^{\prime \prime}\left(T_{t w r}\right)=\frac{9 \times 2^{\frac{3 C_{1}}{T_{t w r} W}} \mu(\ln 2)^{2} C_{1}^{2} C}{T_{t w r}^{3} W^{2}}$ can be obtained.

With $E_{t}^{\prime \prime}\left(T_{t w r}\right) \geq 0, E_{t}$ that is a convex function of $T_{t w r}$ can be known. Then, the problem can be solved with Algorithm 1. The complexities of Algorithm 1 are $\mathcal{O}\left(\mathcal{I}_{d_{1}}+\mathcal{I}_{d_{2}}\right)$ in DT, $\mathcal{O}\left(\mathcal{I}_{d_{3}}+\mathcal{I}_{d_{4}}\right)$ in OWRT, and $\mathcal{O}\left(\mathcal{I}_{d_{5}}\right)$ in TWRT. The $\mathcal{I}_{d_{1}}$ and $\mathcal{I}_{d_{2}}$ are respectively required number of iterations for $T_{1}$ and $T_{2}$ in DT. The $\mathcal{I}_{d_{3}}$ and $\mathcal{I}_{d_{4}}$ are respectively required number of iterations for $T_{1}$ and $T_{2}$ in OWRT, and the $\mathcal{I}_{d_{5}}$ is the required number of iterations for $T_{t w r}$ in TWRT. In order to get the OTT, the complexity can be modeled in polynomial form in terms of the number of variables and constraints with the functions (19a), (23a), (23b), and (26a). Then, for each OTT, it mainly needs one derivation in step 3 and its complexity is $\mathcal{O}(1)$.

Remark 2: There is a pity that the analytic expression of OTT cannot be obtained for the existence of exponential terms of TT, and that is why we use Algorithm 1 to get the OTT in this section.

\subsection{Asymmetry and asymmetry transmission tasks}

In this subsection, the influences of asymmetry and asymmetry transmission tasks at two directions are given. With $\beta$ to show the transmission task distribution factor at two directions, Proposition 5 can be obtained.

Proposition 5 When $C_{T}$ is constant and different nodes' CPs for transmit, receive, and idle are the same, $\beta$ influences $\eta_{t}$ and the maximum $\eta_{t}$ can be obtained when $\beta=0$. However, $\beta$ does not influence $\eta_{d}$ and $\eta_{o}$.

Proof: Please see the Appendix: Proof of Proposition 5.

The proof is completed.

\section{Maximize energy efficiency}

Thirdly, we try to maximize $C_{T}$ and minimize $E_{T}$ simultaneously to maximize $\eta$ with the proposed optimal algorithms. Maximizing $E E$ is a more scientific method to achieve green communication, and many communication techniques have been studied to improve the EEs of different wireless communication systems, i.e., the MIMO system [1, 32]. Thus, the discussion about maximizing $C_{T}$ and minimizing $E_{T}$ simultaneously to maximize $\eta$ maybe also can effectively improve the EE of the MIMO system. 
Actually, the proposed optimal algorithms in this section can also be used in the communication systems when the multiple optimal variables cannot be obtained simultaneously [40]. The usage of the proposed optimal algorithms in the communication systems with multiple optimal variables is only to try to show its application in the existing works, and the further detailed discussions about whether the usage can improve the performance of the related systems can be studied in the further work. This is the same as Sections 4 and 5 with the application of their solutions in the related systems.

\subsection{Energy-efficiency maximization optimal algorithms}

In this subsection, EE maximization optimal algorithms are given. First, maximizing $\eta_{t}$ is given for we have proved in Remark 1: that the OTT in it is always $T_{t w r}=T_{t}$ when the TPs are not functions of TT. Then maximizing $\eta_{t}$ problem is only concerned with TPs. With (3) and the optimization constraints of (24), maximizing $\eta_{t}$ problem can be given as

$$
\begin{aligned}
\max _{P, P_{r}} & \eta_{t}=\frac{W \log _{2}\left(1+\gamma_{t_{1}}+\gamma_{t_{2}}+\gamma_{t_{1}} \gamma_{t_{2}}\right)}{\mu\left(2 P+P_{r}\right)+P_{t}^{c}} \\
\text { s.t. } 0 & \leq\left\{P, P_{r}\right\} \leq P_{t}^{\max }, \gamma_{t_{1}} \geq 2^{\frac{3 C_{1}}{T_{t} W}}-1 \text {, and } \gamma_{t_{2}} \geq 2^{\frac{3 C_{2}}{T_{t} W}}-1,
\end{aligned}
$$

where $P_{t}^{c}=3\left(P_{t_{c 1}}+P_{t_{c 2}}+P_{t_{c 3}}\right)$. At the same time, with $P_{1}=P_{2}=P, o_{1}=o_{2}=$ 0.5 in TWRT [31], $\zeta_{1}$, and $\zeta_{2}, \gamma_{t_{1}}=P\left|h_{3}\right|^{2}+\frac{P P_{r}\left|h_{1}\right|^{2}\left|h_{2}\right|^{2}}{\left(\left|h_{2}\right|^{2}+\left|h_{1}\right|^{2}\right) P_{r}+2 P\left|h_{1}\right|^{2}}$ and $\gamma_{t_{2}}=P\left|h_{3}\right|^{2}+$ $\frac{P P_{r}\left|h_{1}\right|^{2}\left|h_{2}\right|^{2}}{\left(\left|h_{2}\right|^{2}+\left|h_{1}\right|^{2}\right) P_{r}+2 P\left|h_{2}\right|^{2}}$ can be obtained.

In (27), $\eta_{t}$ is non-convex in $\left(P, P_{r}\right)$ for the numerator of $\eta_{t}$ is concave and the denominator of $\eta_{t}$ is linear with respect to $P$ and $P_{r}$, respectively. At the same time, $\eta_{t}$ is differentiable; thus, it is pseudo-concave in terms of $P$ and $P_{r}$, respectively. Since for any optimization problems, we can first optimize over some of the variables and then over the remaining ones [40]. Then, we can divide it into two sub-optimization problems which optimizing $P$ and $P_{r}$ in alternative. The pseudo-concave properties can be proved with Hessians of them, i.e., $\nabla^{2} \eta_{t}(P) \leq 0$ and $\nabla^{2} \eta_{t}\left(P_{r}\right) \leq 0$.

For the pseudo-concave properties of $\eta_{t}$, it can be seemed as $\frac{f(x)}{g(x)}$, where $f(x)$ is concave and $g(x)$ is linear. Define the function $F(\psi)$ as $F(\psi)=\max _{x \in S}\{f(x)-\psi g(x)\}$ with continuous and positive $f, g$, and compact $S$, then $F(\psi)$ is convex with respect to $\psi$; also, it is strictly decreasing and it has a unique root $\psi^{*}$. With it, the problem of finding $F(\psi)$ can be solved with convex optimization approaches and it is shown that the problem of maximizing $\frac{f(x)}{g(x)}$ is equivalent to finding $\psi^{*}[36]$. At the same time, since the original problem has been divided into two sub-optimization problems, it can be solved one-by-one. For each $x$, Dinkelbach's algorithm is employed for its properties in solving non-convex fractional programming problems. Dinkelbach's algorithm can be summarized as Algorithm 2, where the superscript ( $n$ ) denotes the iteration number.

Algorithm 3 leads to global optimal values of each pseudo-concave function. In this regard, for TWRT, firstly, with $P^{(n)}$, Algorithm 2 is adopted in order to find $P_{r}^{(n+1)}$. Secondly, with known $P_{r}^{(n+1)}, P^{(n+1)}$ is computed. Consequently, the one-by-one optimal algorithm is required in order to optimize $P$ and $P_{r}$, simultaneously. Algorithm 3 presents the one-by-one optimal alternating procedure which updates the optimization parameters until convergence.

Second, maximizing $\eta_{d}$ and $\eta_{o}$ problems are given. With $C_{d}\left(P_{1}, P_{2}, T_{1}, T_{2}\right)$, $E_{d}\left(P_{1}, P_{2}, T_{1}, T_{2}\right)$, and the optimization constraints of (17), maximizing $\eta_{d}$ problem can 

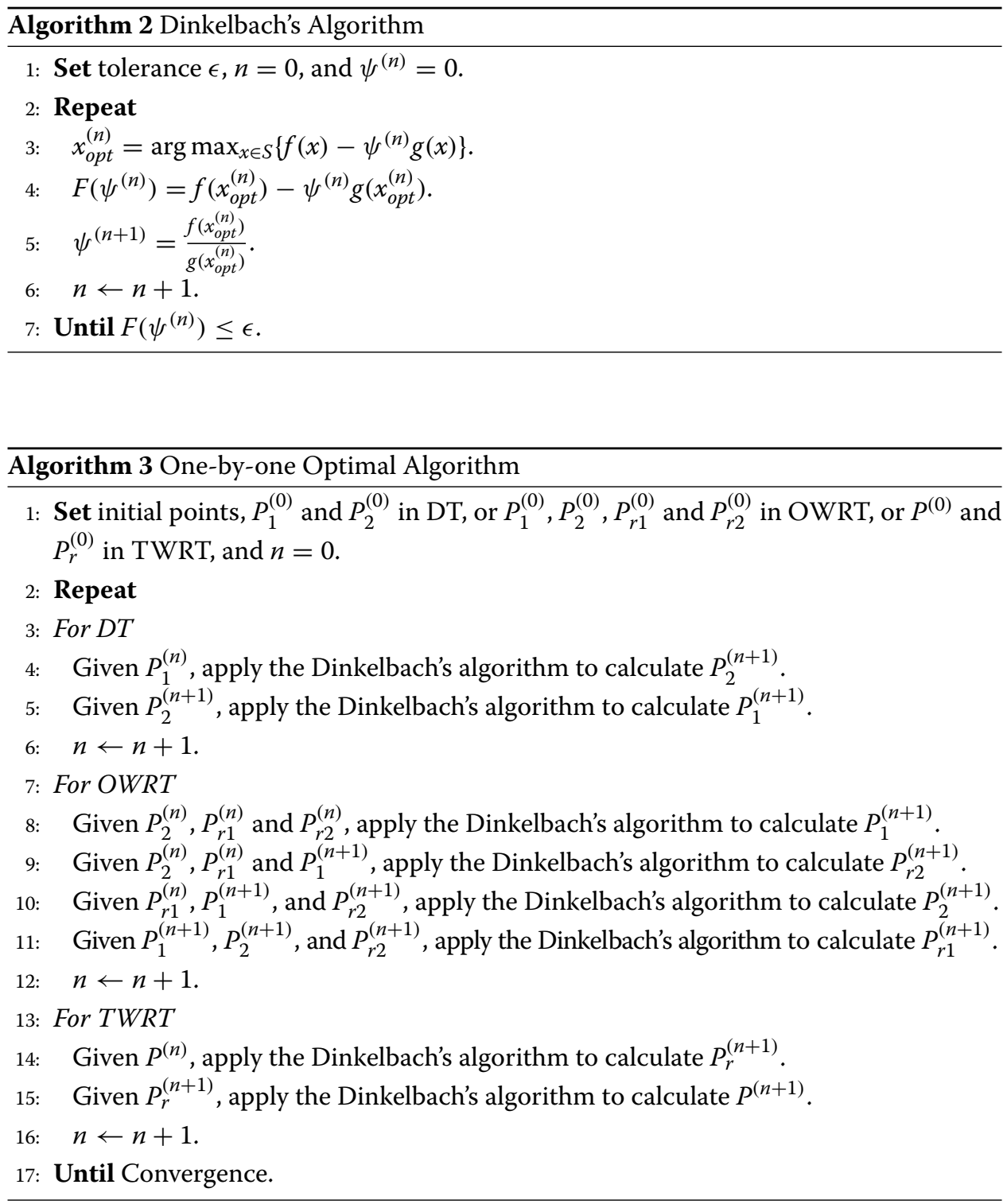

be given as

$$
\begin{aligned}
\max _{P_{1}, P_{2}, T_{1}, T_{2}} \eta_{d}=\frac{C_{d}\left(P_{1}, P_{2}, T_{1}, T_{2}\right)}{E_{d}\left(P_{1}, P_{2}, T_{1}, T_{2}\right)} \\
\text { s.t. } 0 \leq\left\{P_{1}, P_{2}\right\} \leq P_{t}^{\max }, 0 \leq\left\{T_{1}, T_{2}\right\}, T_{1}+T_{2} \leq T_{t}, C_{d_{1}} \geq C_{1} \text {, and } C_{d_{2}} \geq C_{2} .
\end{aligned}
$$

Observing (28), it can be found that the OTT and OTP cannot be obtained simultaneously for the TT and TPs have multiplicative terms. But we also can use Algorithms 2 and 3 to get them one-by-one. However, in practice, there is an optimization order problem with one-by-one optimization method especially for TT and TPs are all existed in it. To simplify the theoretical analysis, we let $T_{1}=T_{2}=T=\frac{1}{2} T_{t}$ in DT and OWRT for it has been suggested in [23] that with high transmission tasks the system will use entail 
block for transmission, then (28) can be changed into

$$
\begin{aligned}
& \max _{P_{1}, P_{2}} \eta_{d}=\frac{W \log _{2}\left(1+\gamma_{d_{1}}+\gamma_{d_{2}}+\gamma_{d_{1}} \gamma_{d_{2}}\right)}{\mu\left(P_{1}+P_{2}\right)+P_{d}^{c}} \\
& \text { s.t. } \frac{2^{\frac{2 C_{1}}{T_{t} W}}-1}{\left|h_{3}\right|^{2}} \leq P_{1} \leq P_{t}^{\max }, \frac{2^{\frac{2 C_{2}}{T_{t} W}}-1}{\left|h_{3}\right|^{2}} \leq P_{2} \leq P_{t}^{\max },
\end{aligned}
$$

where $\gamma_{d_{1}}=P_{1}\left|h_{3}\right|^{2}, \gamma_{d_{2}}=P_{2}\left|h_{3}\right|^{2}$, and $P_{d}^{c}=P_{d_{c 1}}+P_{d_{c 2}}$. Following the same analysis method as TWRT, and with $\nabla^{2} \eta_{d}\left(P_{1}\right) \leq 0, \nabla^{2} \eta_{d}\left(P_{2}\right) \leq 0$, (29a) which are respectively pseudo-concave in terms of $P_{1}$ and $P_{1}$ can be proved. Then, (29) can be solved with Algorithms 2 and 3 as TWRT.

With $T_{1}=T_{2}=\frac{1}{2} T_{t}=T$, and the optimization constraints of (20), the maximizing $\eta_{o}$ can be given as

$$
\begin{aligned}
\max _{P_{1}, P_{2}, P_{r 1}, P_{r 2}} \eta_{o} & =\frac{W \log _{2}\left(1+\gamma_{o_{1}}+\gamma_{o_{2}}+\gamma_{o_{1}} \gamma_{o_{2}}\right)}{\mu\left(P_{1}+P_{2}+P_{r 1}+P_{r 2}\right)+P_{o}^{c}} \\
\text { s.t. } 0 & \leq\left\{P_{1}, P_{2}, P_{r 1}, P_{r 2}\right\} \leq P_{t}^{\max }, \gamma_{o_{1}} \geq 2^{\frac{4 C_{1}}{T_{t} W}}-1, \text { and } \gamma_{o_{2}} \geq 2^{\frac{4 C_{2}}{T_{t} W}}-1,
\end{aligned}
$$

where $P_{o}^{c}=P_{o_{c 1}}+P_{o_{c 2}}$. At the same time, with $A_{1}$ and $A_{2}, \gamma_{o_{1}}=P_{1}\left|h_{3}\right|^{2}+\frac{P_{1} P_{r 2}\left|h_{1}\right|^{2}\left|h_{2}\right|^{2}}{\left(P_{r 2}\left|h_{2}\right|^{2}+P_{1}\left|h_{1}\right|^{2}\right)}$ and $\gamma_{o_{2}}=P_{2}\left|h_{3}\right|^{2}+\frac{P_{2} P_{r 1}\left|h_{1}\right|^{2}\left|h_{2}\right|^{2}}{\left(P_{r 1}\left|h_{1}\right|^{2}+P_{2}\left|h_{2}\right|^{2}\right)}$ can be obtained. Following the same analysis method as TWRT, and with $\nabla^{2} \eta_{o}\left(P_{1}\right) \leq 0, \nabla^{2} \eta_{o}\left(P_{r 2}\right) \leq 0$, (30a) which are respectively pseudoconcave in terms of $P_{1}$ and $P_{r 2}$ can be proved. At the same time, with $\nabla^{2} \eta_{o}\left(P_{2}\right) \leq 0$ and $\nabla^{2} \eta_{o}\left(P_{r 1}\right) \leq 0$, (30a) which are respectively pseudo-concave in terms of $P_{2}$ and $P_{r 1}$ can be proved. Then, (30) can also be solved with Algorithms 2 and 3 as TWRT.

\subsection{Complexity analysis}

In this subsection, the complexity analysis of the optimal algorithms is given. To analyze the computational complexity of Algorithm 3, we should note that Algorithm 3 employs Algorithm 2. But it also should be noted that the convergence rate of Algorithm 2 is independent of the complexity of finding $x_{\mathrm{opt}}^{(n)}$ for its super linear convergence. As the problems of finding $x_{\mathrm{opt}}^{(n)}$ in Algorithm 3 are convex, their complexity can be modeled in polynomial form in terms of the number of variables and constraints. With these properties, we analyze the complexity of Algorithm 3 for DT, OWRT, and TWRT, respectively.

The complexities for step 4 and step 5 are $\mathcal{O}(1)$, and the total complexity of one iteration of Algorithm 3 for DT is $\mathcal{O}\left(\mathcal{I}_{d_{6}}+\mathcal{I}_{d_{7}}\right)$, where $\mathcal{I}_{d_{6}}$ and $\mathcal{I}_{d_{7}}$ are respectively the required number of iterations for step 4 and step 5 . The complexities from step 8 to step 11 are $\mathcal{O}(1)$, and the total complexity of one iteration of Algorithm 3 for OWRT is $\mathcal{O}\left(\mathcal{I}_{d_{8}}+\right.$ $\mathcal{I}_{d_{9}}+\mathcal{I}_{d_{10}}+\mathcal{I}_{d_{11}}$ ), where $\mathcal{I}_{d_{8}}, \mathcal{I}_{d_{9}}, \mathcal{I}_{d_{10} 0}$, and $\mathcal{I}_{d_{11}}$ are respectively the required number of iterations for step 8 , step 9 , step 10, and step 11 . The complexities for step 14 and step 15 are $\mathcal{O}(1)$, and the total complexity of one iteration of Algorithm 3 for TWRT is $\mathcal{O}\left(\mathcal{I}_{d_{12}}+\mathcal{I}_{d_{13}}\right)$, where $\mathcal{I}_{d_{12}}$ and $\mathcal{I}_{d_{13}}$ are respectively the required number of iterations for step 14 and step 15 .

\section{Simulation results}

In this section, simulations are conducted to confirm the validity of theoretical analysis. The simulation parameters are given as Table 2. Considering the Rayleigh fading channel, $\left|h_{3}\right|^{2}=1,\left|h_{1}\right|^{2}=d^{-\alpha}$, and $\left|h_{2}\right|^{2}=(1-d)^{-\alpha}$, where $d \in(0,1)$ are also used in the simulations [31]. Some of the parameters in the simulations are set to a constant, i.e., 
Table 2 Simulation parameters

\begin{tabular}{lll}
\hline Symbol & Definition & Values \\
\hline$T_{t}$ & Block duration & $5 \mathrm{~ms}$ \\
$\mu$ & Power amplifier efficiency & 1.35 \\
$\alpha$ & Path loss attenuation factor & $2-4$ \\
$\beta$ & Transmission task ratio & $0-1$ \\
$P_{l}^{c t}$ & Circuit power with transmit & 0 to hundreds of $\mathrm{mW}$ \\
$P_{l}^{c r}$ & Circuit power with receive & 0 to hundreds of $\mathrm{mW}$ \\
$P_{l}^{c i}$ & Circuit power with idle & 0 to hundreds of $\mathrm{mW}$ \\
$P_{l}^{s i c}$ & Circuit power with SIC & 0 to hundreds of $\mathrm{mW}$ \\
\hline
\end{tabular}

$d=0.5$ in RT, $o_{1}=o_{2}=0.5$ in TWRT, $\alpha=4, \beta=0.5$, and $T^{\prime}=0$, unless otherwise specified.

\subsection{Maximize sum throughput}

In this subsection, EEs with maximizing $C_{T}$ are given. At the same time, TPs are equally allocated. In Fig. 2, EEs with and without idle state are given which correspond to $T^{\prime}=1 \mathrm{~ms}$ and $T^{\prime}=0$, respectively. The with and without idle state corresponds to " $w$ " and " $w / o$ " in Fig. 2, respectively. From it, we can find that EEs when $T^{\prime}=1 \mathrm{~ms}$ are lower than those when $T^{\prime}=0$, which corresponds to Propositions 1 and 2 that the OTT both in DT and RT to maximize EE is $T^{\prime}=0$.

In Fig. 3, EEs with different $o_{1}$ in TWRT are given. With it, two results can be found: (i) EE of TWRT when $o_{1}=0.1$ is the same as when $o_{1}=0.9$, and EE of TWRT when $o_{1}=0.25$ is the same as when $o_{1}=0.75$; (ii) EE of TWRT when $o_{1}=0.5$ is the maximum. These have been proved in Proposition 4 for $f^{\prime \prime}\left(o_{1}\right)<0$ and $f^{\prime}(0.5)=0$, which means the EE of TWRT is the maximum when $o_{1}=0.5$. In Fig. 3, EEs of DT and OWRT, and EE of relay transmission with none of DL (RT-NDL) which corresponding to one-way relay

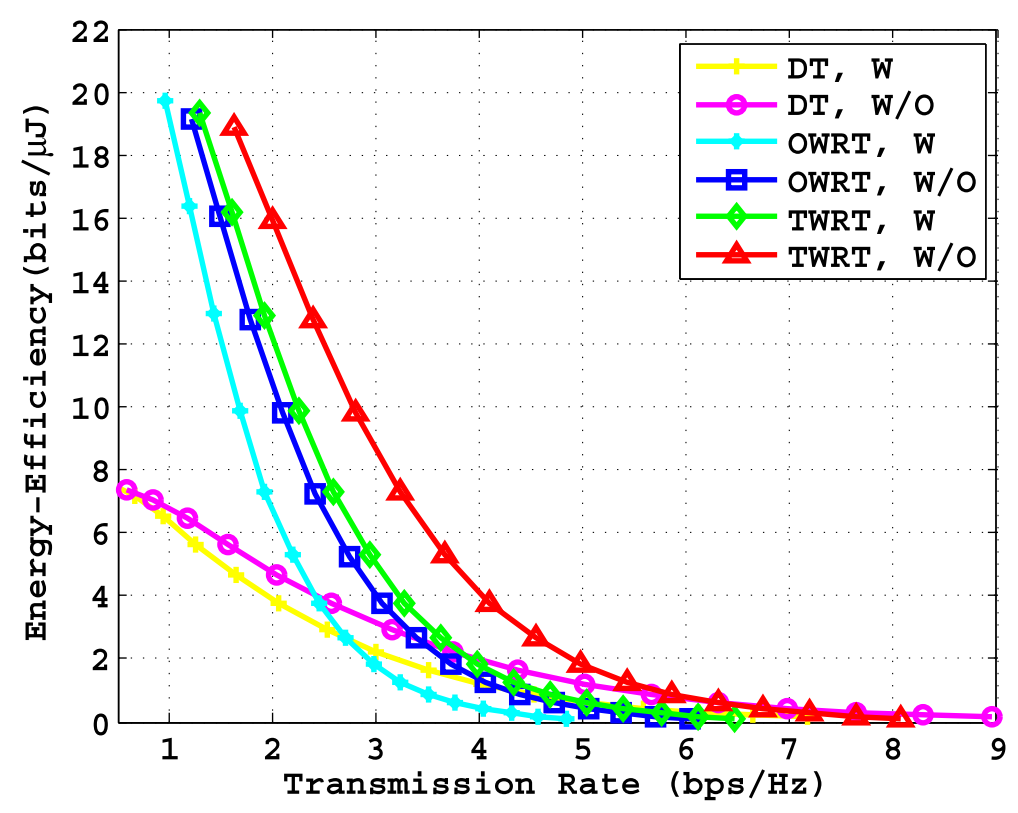

Fig. 2 EEs with and without idle state 


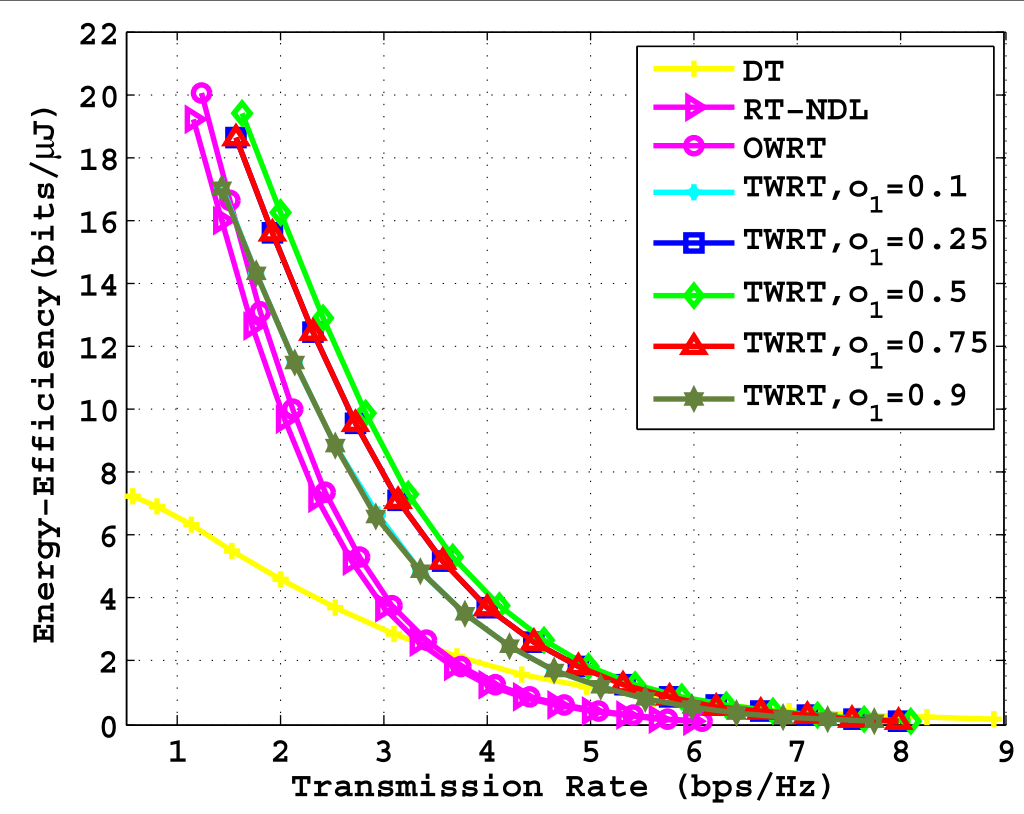

Fig. 3 EEs with different $o_{1}$

system in [23] are also given. From it, we can know that EEs with DLs in RT are higher than those of RT-NDL, which shows the effectiveness of DLs in RT for its SE performance gain.

In Fig. 4, EEs of RT with different $d$ are given. In Fig. 4a, it can be found that EE of OWRT is the maximum when relay node lies in the middle of two source nodes, which has been proved in Proposition 3 for in such case $\gamma_{o_{1}}$ is the maximum. In Fig. 4b, it can be found that the smaller the $d$ the higher the EE of TWRT, which also has been proved in Proposition 4 for in such case $\gamma_{t}$ is bigger.

In Fig. 5, outage probabilities are given. The simulation results of outage probabilities are obtained with (6), (11) and (16). From Fig. 5, it can be found that outage performance of TWRT is the best and DT is the worst. This is because with the given $C_{t h}$ to avoid disruption, the ST of TWRT is the best and ST of DT is the worst.
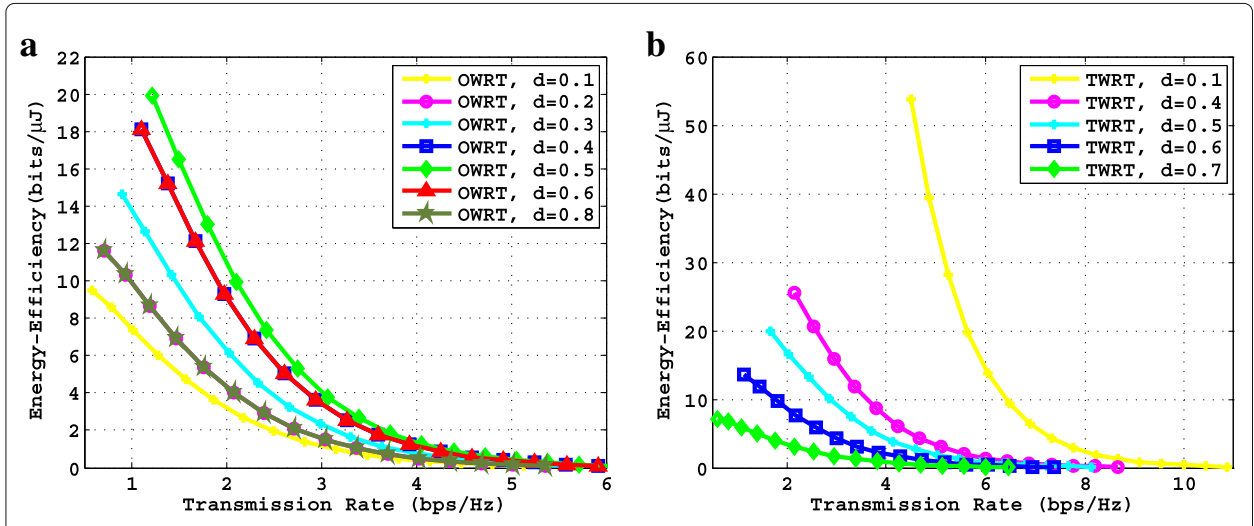

Fig. 4 EEs with different $d$. a OWRT. b TWRT 


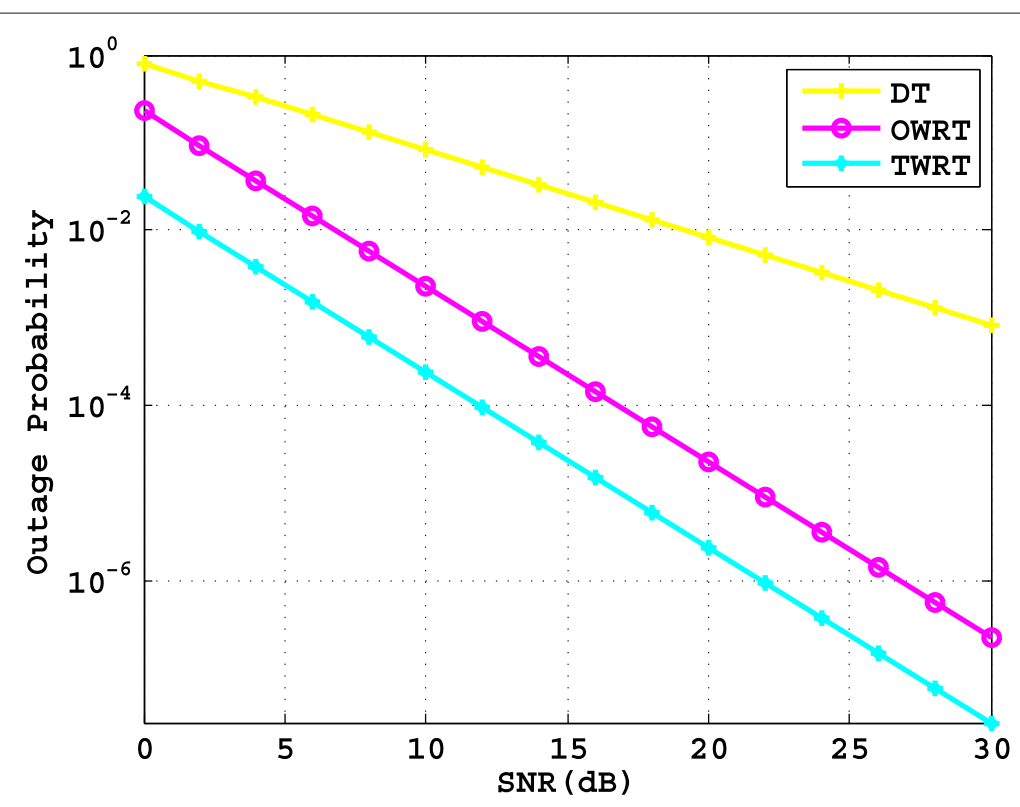

Fig. 5 Outage probability

\subsection{Minimize total energy consumption}

In this subsection, EEs with minimizing $E_{T}$ are given. At the same time, TPs are optimally allocated. In Fig. 6, EEs when $T^{\prime}=1 \mathrm{~ms}$ and $T^{\prime}=0$ are given. The $T^{\prime}=1 \mathrm{~ms}$ corresponds to the DT, OWRT, and TWRT with idle state, and it means the system can transfer into idle state when the transmission tasks have been completed. The $T^{\prime}=0 \mathrm{~ms}$ corresponds to the DT, OWRT, and TWRT without idle state, and it means the system always uses the entail block duration for transmission. The with and without idle states correspond to " $w$ " and "w/o" in the Fig. 6, respectively. From Fig. 6, the following results can be found: (i)

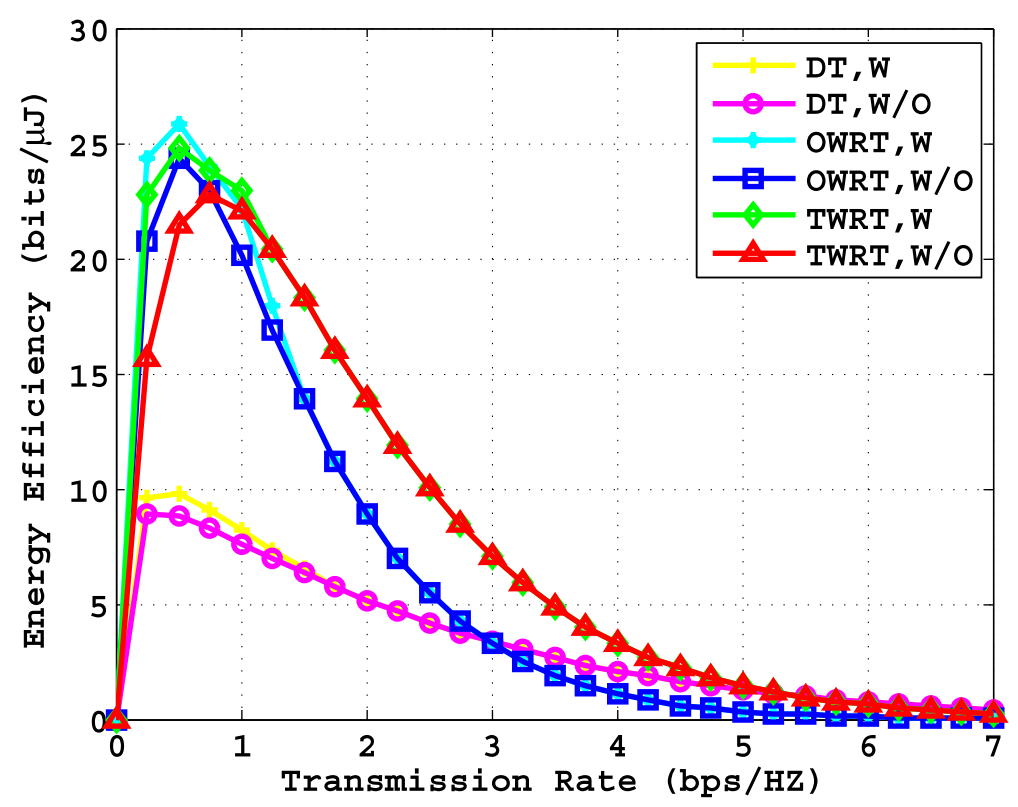

Fig. 6 EEs with and without idle state 
EEs when $T^{\prime}=0$ are lower than those when $T^{\prime}=1 \mathrm{~ms}$ when transmission rate is low; (ii) EEs when $T^{\prime}=0$ are equal to those when $T^{\prime}=1 \mathrm{~ms}$ when transmission rate is high. This is because when the transmission task is low and it has been completed, the without idle mode still uses entail block duration $T_{t}$ for transmission. In such case, TPs and other CPs, such as transmit and receive CPs, keep consuming. However, the with idle mode changes to the idle mode. In such case, TPs and other CPs can be saved even with idle CPs increase. However, idle CPs are smaller than TPs and other CPs. Finally, the EEs with idle mode are higher. This shows the necessity of TT optimization. While when the transmission rate is high, both with and without idle modes will use entail $T_{t}$ for transmission, and the EEs of them are the same.

In Fig. 7, EEs with different $\beta$ are given. From Fig. 7, firstly, it can be found that $\beta$ does not influence the EEs of DT and OWRT, but it influences the EE of TWRT. Secondly, it can be found that the bigger the $\beta$, the lower the EE of TWRT. All of these have been proved in Proposition 5. In Fig. 7, EE of RT-NDL is also given. From it, we can know that EE of RT-NDL is the worst in RT even it compares with EE of TWRT when $\beta=0.6$, which also shows the effectiveness of DLs in RT.

Comparing EEs in minimizing $E_{T}$ with maximizing $C_{T}$, two results can be found: (i) EEs are no more a decreasing function. This is because the OTT in maximizing $C_{T}$ is always $T^{\prime}=0$. While in minimizing $E_{T}$, when the transmission tasks have been completed, the transmission models can change into idle state. Then the influences of CPs become more obvious, when $\mathrm{SE}$ is low, the CPs are bigger than TPs and EEs are increasing functions. While when SE is high, TPs are much bigger than CPs, the influences of CPs can be ignored, and EEs are decreasing functions; (ii) EEs of OPA are higher than those of equal power allocation, which shows the significance of OPA.

In Fig. 8, EEs with maximizing $C_{T}$ and minimizing $E_{T}$ simultaneously are also given. From Fig. 8, it can be found that EEs of maximizing $C_{T}$ and minimizing $E_{T}$ simultaneously

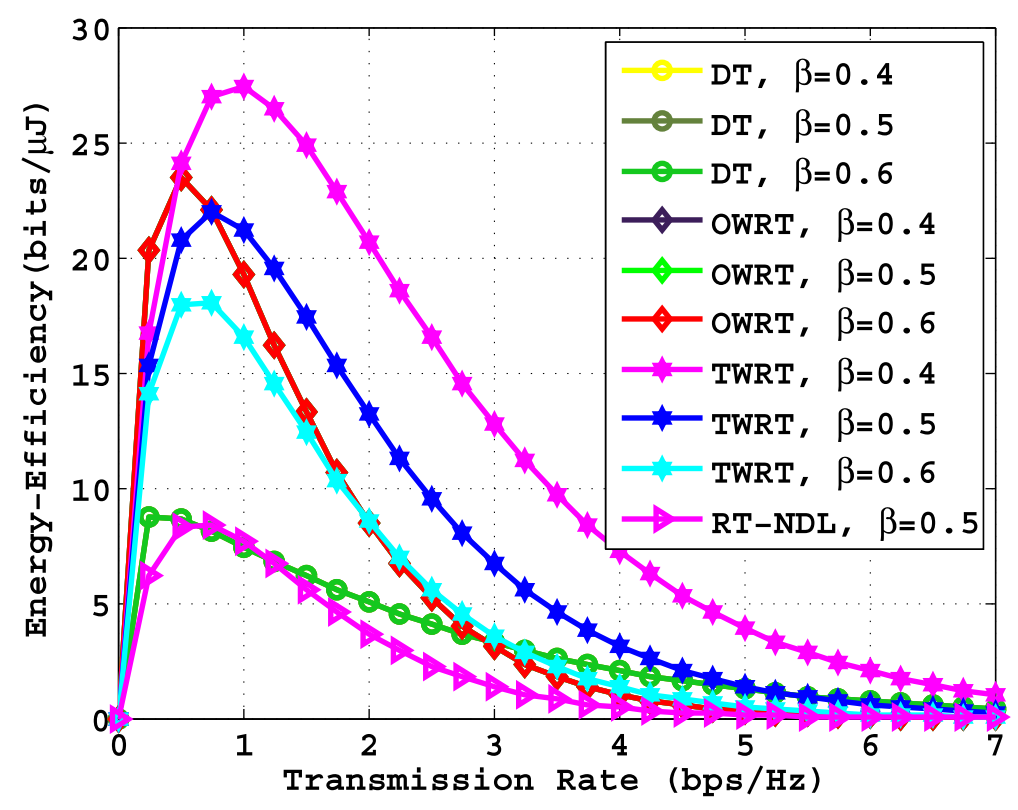

Fig. 7 EEs with different $\beta$ 
are higher than that of only maximizing $C_{T}$ or only minimizing $E_{T}$. This is because the OTPs in it need to consider $C_{T}$ and $E_{T}$, simultaneously. In Fig. 8, EE of RT-NDL which is the worst in RT can also be obtained.

From Figs. 2, 3, 4, 5, 6, 7, and 8, it can be found that among different transmission models, EE of TWRT is almost always the maximum and EE of DT is almost always the minimum. The TWRT has the maximum EE both for relay node's assistance and its time delay advantage. Also, it can be found that EE of RT-NDL is lower than the other RT with DLs. These two phenomenons suggest that relay technique and DLs in RT can improve system's EE.

\section{Conclusion}

In this paper, throughput, energy consumption, and EE with consideration of non-ideal PA efficiency, non-negligible CP consumption, and DLs in RT, through joint optimization of TPs and TT allocation, have been analyzed. The EET with three optimization cases have been given, and several characteristics could be found: (i) with maximizing $C_{T}$ to maximize EE, EEs without idle state are higher, EE of OWRT when relay node is closer to the middle of two source nodes is higher, and EE of TWRT when relay node is closer to node $S_{1}$ and $o_{1}=0.5$ is higher. At the same time, outage probability of TWRT is the best; (ii) with minimizing $E_{T}$ to maximize EE, EEs without idle state are lower when SE is low and transmission tasks ratio only influences the EEs of TWRT; (iii) with Dinkelbach's algorithm and one-by-one optimal algorithm to maximize EE, EEs are higher than that of only maximizing $C_{T}$ or only minimizing $E_{T}$; (iv) relay technique and DLs in RT can improve system's EE. All of these have been verified by the theoretical analysis and the simulations. For the energy harvesting property of SWIPT, the EEs of these three cases with SWIPT can be studied in the further work.

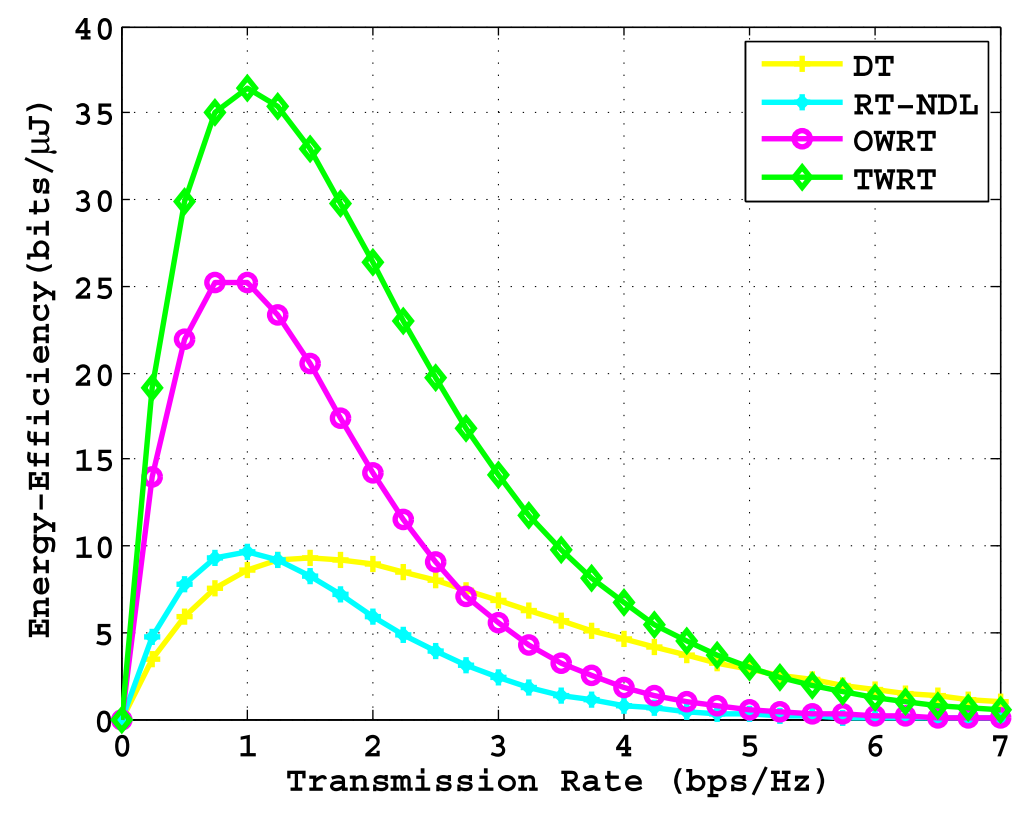

Fig. 8 EEs with maximizing EE 


\section{Appendix}

\section{Proof of Proposition 4}

First, optimal $o_{1}$ and $o_{2}$ are analyzed to maximize $\gamma_{t}$ under the constraints of $o_{1}+o_{2}=1$ and $\left\{o_{1}, o_{2}\right\} \in[0,1]$. With $P_{1}=P_{2}=P_{r 1}=P_{r 2}=P_{r}=P, T^{\prime}=0, \zeta_{1}, \zeta_{2}$, and channel gains, $\gamma_{t_{1}}$ and $\gamma_{t_{2}}$, can be reformulated as

$$
\begin{aligned}
& \gamma_{t_{1}}=P\left(1+\frac{o_{1} d^{-\alpha}(1-d)^{-\alpha}}{o_{1}\left((1-d)^{-\alpha}-d^{-\alpha}\right)+2 d^{-\alpha}}\right) \\
& \gamma_{t_{2}}=P\left(1+\frac{\left(1-o_{1}\right) d^{-\alpha}(1-d)^{-\alpha}}{o_{1}\left((1-d)^{-\alpha}-d^{-\alpha}\right)+d^{-\alpha}+(1-d)^{-\alpha}}\right) .
\end{aligned}
$$

Assuming $P=a, b\left(o_{1}\right)=\frac{o_{1} d^{-\alpha}(1-d)^{-\alpha}}{o_{1}\left((1-d)^{-\alpha}-d^{-\alpha}\right)+2 d^{-\alpha}}$, and $c\left(o_{1}\right)=\frac{\left(1-o_{1}\right) d^{-\alpha}(1-d)^{-\alpha}}{o_{1}\left((1-d)^{-\alpha}-d^{-\alpha}+d^{-\alpha}+(1-d)^{-\alpha}\right.}$, then $\gamma_{t_{1}}=a+a b\left(o_{1}\right), \gamma_{t_{2}}=a+a c\left(o_{1}\right)$, and $\gamma_{t}=\gamma_{t_{1}}+\gamma_{t_{2}}+\gamma_{t_{1}} \gamma_{t_{2}}=a^{2}+2 a+a(a+1)\left(b\left(o_{1}\right)+\right.$ $\left.c\left(o_{1}\right)\right)+a^{2} b\left(o_{1}\right) c\left(o_{1}\right)$ can be obtained. Ignore all the parts that have no relation with $o_{1}$ in $\gamma_{t}$, the problem about optimal $o_{1}$ to maximize $\gamma_{t}$ can be given as

$$
\begin{aligned}
& \max _{o_{1}} f\left(o_{1}\right)=a(a+1)\left(b\left(o_{1}\right)+c\left(o_{1}\right)\right)+a^{2} b\left(o_{1}\right) c\left(o_{1}\right) \\
& \text { s.t. } 0 \leq o_{1} \leq 1 .
\end{aligned}
$$

With $f^{\prime \prime}\left(o_{1}\right)<0$ and $f^{\prime}(0.5)=0, \eta_{t}$ is the maximum when $o_{1}=o_{2}=0.5$ can be obtained for in such case $\gamma_{t}$ is the maximum.

Second, ORP is analyzed to maximize $\gamma_{t}$. With $o_{1}=0.5$, the ORP problem in TWRT can be given as

$$
\begin{aligned}
& \max _{d} g(d)=a(a+1)(b(d)+c(d))+a^{2} b(d) c(d) \\
& \text { s.t. } 0<d<1,
\end{aligned}
$$

where $b(d)=\frac{d^{-\alpha}(1-d)^{-\alpha}}{(1-d)^{-\alpha}+3 d^{-\alpha}}$ and $c(d)=\frac{d^{-\alpha}(1-d)^{-\alpha}}{3(1-d)^{-\alpha}+d^{-\alpha}}$. With $g^{\prime}(d)<0, \gamma_{t}$ is a decreasing function of $d$ that can be obtained, which means when the relay node is near node $S_{1}, \eta_{t}$ is higher for in such case $\gamma_{t}$ is bigger.

\section{Proof of Proposition 5}

It has been shown that the optimization problem of (19) is a convex problem and it can be solved separately; then, the optimal $T_{1}$ and $T_{2}$ in DT should satisfy the Karush-KuhnTucker (KKT) conditions. With $P_{d_{c 1}}=P_{d_{c 2}}=P_{d_{c}}$, then the KKT conditions can be expressed as

$$
\begin{aligned}
& \lambda\left(T_{1}+T_{2}-T_{t}\right)=0, \\
& \frac{\mu 2^{\frac{\beta C_{T}}{T_{1} W}}}{\left|h_{3}\right|^{2}}\left(1-\ln 2 \frac{\beta C_{T}}{T_{1} W}\right)+P_{d_{c 1}}-P_{D c i}=-\lambda, \\
& \frac{\mu 2^{\frac{(1-\beta) C_{T}}{T_{2} W}}}{\left|h_{3}\right|^{2}}\left(1-\ln 2 \frac{(1-\beta) C_{T}}{T_{2} W}\right)+P_{d_{c 2}}-P_{D c i}=-\lambda,
\end{aligned}
$$

where $\lambda$ is the Lagrange multiplier. From (34), it can be found that the left-hand sides of (34b) and (34c) are equal to each other, then the optimal TT satisfies $\frac{\beta C_{T}}{T_{1}}=\frac{(1-\beta) C_{T}}{T_{2}}=$ $R_{d}$. Substituting it into the KKT conditions, it is easy to see that $R_{d}$ is not a function of $\beta$. 
With it, the minimum TEC in DT can further be obtained as

$$
\begin{aligned}
E_{d} & =\frac{\beta C_{T}}{R_{d}}\left(\mu \frac{2^{\frac{R_{d}}{W}}}{\left|h_{3}\right|^{2}}+P_{d_{c}}-P_{D c i}\right)+\frac{(1-\beta) C_{T}}{R_{d}}\left(\mu \frac{2^{\frac{R_{d}}{W}}}{\left|h_{3}\right|^{2}}+P_{d_{c}}-P_{D c i}\right)+T_{t} P_{D c i} \\
& =\frac{C_{T}}{R_{d}}\left(\mu \frac{2^{\frac{R_{d}}{W}}}{\left|h_{3}\right|^{2}}+P_{d_{c}}-P_{D c i}\right)+T_{t} P_{D c i},
\end{aligned}
$$

which is not a function of $\beta$. It is easy to understand intuitively. With the optimal TT, the transmission tasks on each direction and each bit is transmitted with identical data rate $R_{d}$ and identical time duration $1 / R_{d}$. Therefore, the energy consumed by each bit is identical no matter in which direction it is. Then the minimum TEC only depends on the $C_{T}$. The same conclusion can also be obtained in OWRT with the same method. All of these show that $\eta_{d}$ and $\eta_{o}$ have no relation with $\beta$.

In TWRT, (26a) that is a increasing function of $\gamma_{t_{1}}=2^{\frac{3 \beta C_{T}}{T_{t w r} W}}-1$ can be known. When $C_{T}$ and $T_{t w r}$ are known, if $\beta$ is increasing, then TEC is increasing. It shows that $\eta_{t}$ is a decreasing function of $\beta$.

\section{Abbreviations}

EE: Energy efficiency; SE: Spectral efficiency; AF: Amplify-and-forward; EET: Energy-efficient transmission; TP: Transmit power; OPA: Optimal power allocation; SET: Spectral-efficient transmission; TT: Transmit time; DT: Direct transmission; OWRT: One-way relay transmission; TWRT: Two-way relay transmission; OTT: Optimal TT; OTP: Optimal TP; PAs: Power amplifiers; CPs: Circuit powers; DLs: Direct links; RT: Relay transmission; CSI: Channel state information; ORP: Optimal relay position; ST: Sum throughput; TEC: Total energy consumption

\section{Acknowledgements}

Not applicable.

\section{Authors' contributions}

The main contributions of Caixia Cai, Xue-Qin Jiang, and Yuyang Peng were to create the main ideas, perform theoretical analysis, and execute performance evaluation by extensive simulation while Runhe Qiu worked as the advisors to discuss, create, and advise the main ideas and performance evaluations together. The authors read and approved the final manuscript.

\section{Funding}

This research was supported in part by China Scholarship Council and Donghua University Graduate Innovation Fund Program under Grant CUSF-DH-D-2017086.

\section{Availability of data and materials}

Data sharing is not applicable to this article as no datasets were generated or analyzed during the current study.

\section{Competing interests}

The authors declare that they have no competing interests.

\section{Author details}

${ }^{1}$ College of Information Sciences and Technology, Donghua University, Renmin North, Shanghai 201620, People's Republic of China. ${ }^{2}$ Engineering Research Center of Digitized Textile \& Fashion Technology, Ministry of Education, Donghua University, Renmin North, Shanghai 201620, People's Republic of China. ${ }^{3}$ Faculty of Information Technology, Macau University of Science and Technology, Macau 999078, China.

Received: 26 August 2019 Accepted: 14 July 2020

Published online: 31 July 2020

\section{References}

1. J. Wu, S. Rangan, H. Zhang, Green Communications: Theoretical Fundamentals, Algorithms, and Applications. (CRC Press, Boca Raton, 2012)

2. D.-T. Do, Energy-aware two-way relaying networks under imperfect hardware: optimal throughput design and analysis. Telecommun. Syst. J. 62 (2), 449-459 (2015)

3. D.-T. Do, A.-T. Le, B. M. Lee, On performance analysis of underlay cognitive radio-aware hybrid OMA/NOMA networks with imperfect CSI. Elect. 8(7), 819, 1-21 (2019) 
4. D.-T. Do, T. Le, C.-B. Le, B. M. Lee, On exact outage and throughput performance of cognitive radio based non-orthogonal multiple access networks with and without D2D link. Sens. 19(15), 3314, 1-17 (2019)

5. J. Li, S. Dang, M. Wen, X.-Q. Jiang, Y. Peng, H. Hai, Layered orthogonal frequency division multiplexing with index modulation. IEEE Syst. J. 13(4), 3793-3802 (2019)

6. Y. Xin, D. Wang, J. Li, H. Zhu, J. Wang, X. You, Area spectral efficiency and area energy efficiency of massive MIMO cellular systems. IEEE Trans. Veh. Technol. 65(5), 3243-3254 (2016)

7. Y. Zhang, M. Xiao, S. Han, M. Skoglund, W. Meng, On precoding and energy efficiency of full-duplex millimeter-wave relays. IEEE Trans. Wirel. Commun. 18(3), 1943-1956 (2019)

8. A. Li, C. Masouros, Energy-efficient SWIPT: from fully digital to hybrid analog-digital beamforming. IEEE Trans. Veh. Technol. 67(4), 3390-3405 (2018)

9. D.-T. Do, H.-S. Nguyen, A tractable approach to analyzing the energy-aware two-way relaying networks in the presence of co-channel interference. Eura. J. Wirel. Commum. Netw. 2016(271), 1-10 (2016)

10. D.-T. Do, A.-T. Le, NOMA based cognitive relaying: transceiver hardware impairments, relay selection policies and outage performance comparison. Comput. Commun. 146, 144-154 (2019)

11. D.-T. Do, C.-B. Le, B. M. Lee, Robust transmit antenna design for performance improvement of cell-rdge users: approach of NOMA and outage/ergodic capacity analysis. Sens. 19(22), 4907, 1-26 (2019)

12. M. Wen, X. Chen, Q. Li, E. Basar, Y. C. Wu, W. Zhang, Index modulation aided subcarrier mapping for dual-hop OFDM relaying. IEEE Trans. Commun. 67(9), 6012-6024 (2019)

13. B. Zheng, M. Wen, C. X. Wang, X. Wang, F. Chen, J. Tang, F. Ji, Secure NOMA based two-way relay networks using artificial noise and full duplex. IEEE J. Sel. Areas Commun. 36(7), 1426-1440 (2018)

14. S. Wang, Q. Song, X. Wang, A. Jamalipour, Rate and power adaptation for analog network coding. IEEE Trans. Veh. Technol. 60(5), 2302-2313 (2011)

15. H. Gao, T. Lv, X. Sun, H. Yang, J. M. Cioffi, Energy-efficient resource allocation for massive MIMO amplify-and-forward relay systems. IEEE Acce. 4, 2771-2787 (2016)

16. Q. Liu, T. Lv, Z. Lin, Energy-efficient transmission design in cooperative relaying systems using NOMA. IEEE Commun. Lett. 22(3), 594-597 (2018)

17. L. Li, C. Dong, L. Wang, L. Hanzo, Spectral-efficient bidirectional decode-and-forward relaying for full-duplex communication. IEEE Trans. Veh. Technol. 65(9), 7010-7020 (2016)

18. B. Klaiqi, X. Chu, J. Zhang, Energy-and spectral-efficient adaptive forwarding strategy for multi-hop device-to-device communications overlaying cellular networks. IEEE Trans. Wirel. Commun. 17(9), 5684-5699 (2018)

19. L. Qian, Y. Wu, J. Wang, W. Zhang, Energy-efficient distributed user scheduling in relay-assisted cellular networks. IEEE Trans. Wirel. Commun. 15(6), 4060-4073 (2016)

20. C. Sun, C. Yang, in Proc. 2012 IEEE International Conference on Communications (ICC), Energy efficiency comparison among direct, one-way and two-way relay transmission, (Ottawa, 2012), pp. 4288-4293. https://doi.org/10.1109/icc. 2012.6363882

21. J. Xu, R. Zhang, Throughput optimal policies for energy harvesting wireless transmitters with non-ideal circuit power. IEEE J. Sel. Areas Commun. 32(2), 322-332 (2014)

22. Q. Cui, Y. Zhang, W. Ni, M. Valkama, R. Janti, Energy efficiency maximization of full-duplex two-way relay with non-ideal power amplifiers and non-negligible circuit power. IEEE Trans. Wirel. Commun. 16(9), 6264-6278 (2017)

23. C. Sun, C. Yang, Energy efficiency analysis of one-way and two-way relay systems. Eura. J. Wirel. Commum. Netw. 2012(46), 1-18 (2012)

24. C. Li, P. Liu, C. Zou, F. Sun, J. M. Cioffi, L. Yang, Spectral-efficient cellular communications with coexistent one- and two-hop transmissions. IEEE Trans. Veh. Technol. 65(9), 6756-6772 (2016)

25. Z. He, X. Zhang, Y. Bi, W. Jiang, Y. Rong, Optimal source and relay design for multiuser MIMO AF relay communication systems with direct links and imperfect channel information. IEEE Trans. Wirel. Commun. 15(3), 2025-2038 (2016)

26. Y. Li, Z. Zheng, Y. Li, G. Cheng, Energy efficiency optimisation for AF relay networks with direct transmissions. Elect. Lett. 51(4), 366-368 (2015)

27. C. Cai, R. Qiu, Jiang X.-Q., in Proc 2020 IEEE International Conference on Artificial Intelligence in Information and Communication (ICAIIC), Optimal resources allocation of bidirectional relay communication, (Fukuoka, 2020), pp. 1-5. https://doi.org/10.1109/icaiic48513.2020.9065272

28. C. Cai, R. Qiu, X.-Q. Jiang, Design and optimization for energy-efficient full-duplex transmission with direct links, early access (2020). https://doi.org/10.1109/tgen.2020.2979244

29. Y. Li, X. Zhu, C. Liao, C. Wang, B. Cao, Energy efficiency maximization by jointly optimizing the positions and serving range of relay stations in cellular networks. IEEE Trans. Veh. Technol. 64(6), 2551-2560 (2015)

30. C. Cai, R. Qiu, Energy-efficient cooperative two-hop amplify-and-forward relay protocol in cognitive radio networks. IET Commum. 10(16), 2135-2142 (2016)

31. M. Ju, I. Kim, Relay selection with ANC and TDBC protocols in bidirectional relay networks. IEEE Trans. Commun. 58(12), 3500-3511 (2010)

32. E. Bjornson, L. Sanguictti, M. Debbah, Deploying dense networks for maximal energy efficiency: small cells meet massive MIMO. IEEE J. Sel. Areas. Commun. 34(4), 832-847 (2016)

33. M. Yang, J. Xia, Y. Guo, A. Zhu, Highly efficient broadband continuous inverse class-F power amplifier design using modified elliptic low-pass filtering matching network. IEEE Trans. Micro. Theo. Tech. 64(5), 1515-1525 (2016)

34. Q. Li, Q. Zhang, J. Qin, Beamforming in non-regenerative two-way multi-antenna relay networks for simultaneous wireless information and power transfer. IEEE Trans. Wirel. Commun. 13(10), 5509-5520 (2014)

35. M. S. K. Lau, W. Yue, L. Ping, Equal power allocation of IDMA systems: feasibility, optimality, and throughput. IEEE Trans. Wirel. Commun. 8(2), 746-756 (2009)

36. A. Zappone, E. Jorswieck, Energy efficiency in wireless networks via fractional programming theory. Found. Trends Commun. Inf. Theory. 11(3-4), 185-396 (2015)

37. Y. Zhang, Z. Yang, Y. Feng, S. Yan, Performance analysis of cooperative relaying systems with power-domain non-orthogonal multiple access. IEEE Acce. 6, 39839-39848 (2018) 
38. J. N. Laneman, D. N. C. Tse, G. W. Wornell, Cooperative diversity in wireless networks: efficient protocols and outage behavior. IEEE Trans. Inf. Theory. 50(12), 3062-3080 (2004)

39. S. Boyd, L. Vandenberghe, Convex Optimization. (Cambridge Univ, Cambridge, 2003)

40. J. Xu, L. Qiu, Energy efficiency optimization for MIMO broadcast channels. IEEE Trans. Wirel. Commun. 12(2), 690-701 (2013)

\section{Publisher's Note}

Springer Nature remains neutral with regard to jurisdictional claims in published maps and institutional affiliations.

\section{Submit your manuscript to a SpringerOpen ${ }^{\circ}$} journal and benefit from:

- Convenient online submission

- Rigorous peer review

- Open access: articles freely available online

- High visibility within the field

- Retaining the copyright to your article

Submit your next manuscript at $\gg$ springeropen.com 